\title{
Modeling and Analysis of Origami Structures with Smooth Folds
}

\author{
Edwin A. Peraza Hernandez ${ }^{\mathrm{a}}$, Darren J. Hartl ${ }^{\mathrm{a}, *}$, Ergun Akleman ${ }^{\mathrm{b}}$, Dimitris C. Lagoudas ${ }^{\mathrm{a}}$ \\ ${ }^{a}$ Department of Aerospace Engineering, Texas A $6 M$ University, College Station, TX 77843-3141 \\ ${ }^{b}$ Visualization Department, Texas A\&BM University, College Station, TX 77843-3137
}

\begin{abstract}
Origami has the potential to impact numerous areas of design and manufacturing. Modeling and analysis of origami structures allow for the understanding of their behavior and the development of computational tools for their design. Most available origami models are limited to the idealization of folds as creases of zeroth-order geometric continuity, which is not proper for origami structures having non-negligible fold thickness or with maximum curvature at the folds restricted by material limitations. Structural analysis of origami sheets having creased folds requires further idealizations of the fold mechanical response such as the representation of the folds as torsional springs. In view of this, a novel model analogous to that for rigid origami is presented in this work for origami structures having folds of non-zero surface area that exhibit higher-order geometric continuity (termed smooth folds). This origami model allows for a proper structural analysis of origami sheets using plate or shell representations for the folds. The shape formulation of the smooth folds and the kinematic constraints on their associated shape variables are presented. Modeling of origami structures with smooth folds exhibiting elastic behavior is performed by determining the configuration of the structure that minimizes its total potential energy subject to the derived kinematic constraints. The presented results show that the structural response determined using the proposed model is in good agreement with both experiments and higher-fidelity finite element analyses.
\end{abstract}

Keywords:

origami, folding, smooth folds, modeling, elastic

\section{Introduction}

Traditionally, in origami a goal shape is achieved exclusively by folding an initially planar sheet of negligible thickness. In this context, a fold is any deformation of the sheet such that the in-surface distance between any two points in the sheet is invariant and self-intersection does not occur [1]. Over the past four decades, there has been an increasing attention from the mathematics, architecture, science, and engineering communities given to theoretical models and computational design tools for origami $[2,3]$. Engineering advantages of origami-inspired structures include compact storage/deployment capability [4], the potential for reconfigurability [5], and a reduction in manufacturing complexity [6], among others [7, 8].

Rigid origami is the special case of origami for which the planar faces bounded by the folds and the sheet boundary undergo only rigid deformations, i.e. these faces are neither bent nor stretched [10]. An example of rigid origami is shown in Figure 2(a). Rigid origami has been studied by several researchers in the past and the subject remains active [11]. For example, it has been recently utilized for the design of pop-up mechanisms $[12,13]$ and deployable structures $[14,15]$.

*darren.hartl@tamu.edu
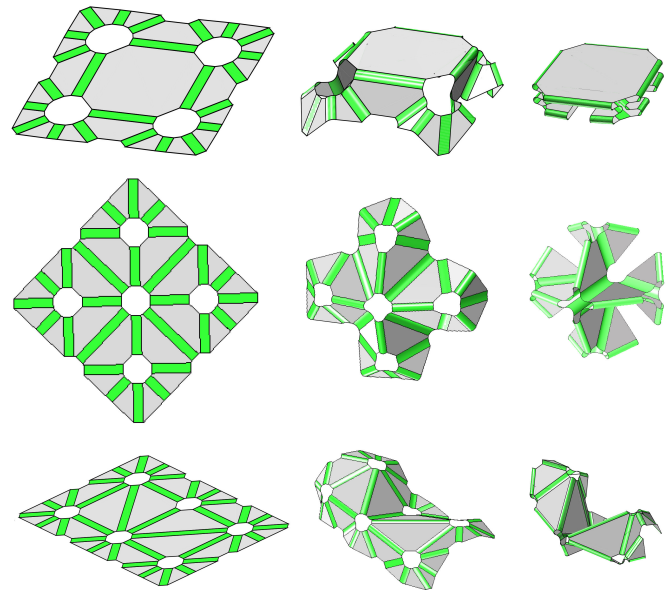

Figure 1: Examples of valid configurations for origami structures with smooth folds. These examples are based on kinematic simulation [9]. The model presented in this paper extends beyond kinematics by considering mechanical equilibrium and material constitutive behavior and allows for a thorough physics-based analysis of origami structures with smooth folds.

Mathematical modeling and simulation of rigid origami structures allows for the understanding of their behavior and the development of computational tools for their de- 


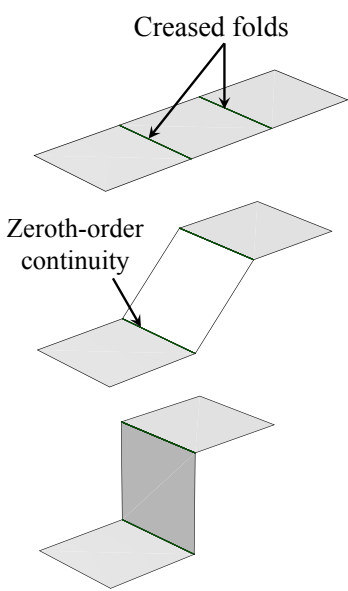

(a)

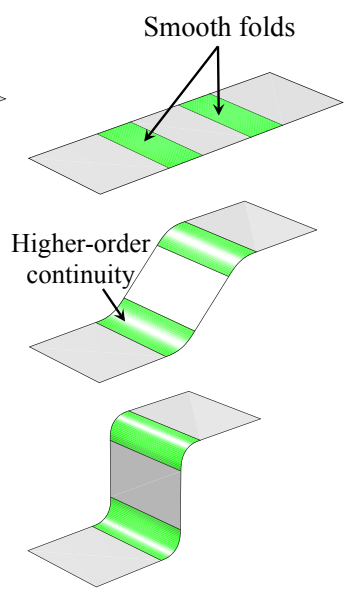

(b)
Figure 2: (a) A sheet with creased folds of zeroth-order geometric continuity $\left(G^{0}\right)$. (b) A sheet with smooth folds of non-zero surface area and higher-order geometric continuity $\left(G^{n}\right)$.

sign. Various approaches have been utilized for the simulation of rigid origami [10]. For example, truss representations [16] have been considered where the faces of the sheet are triangulated, each fold or boundary edge endpoint is represented by a truss joint, and each fold and boundary edge is represented by a truss member. Configurations in which the truss joint displacements do not cause elongations on the truss members [17] represent valid rigid origami configurations.

Alternatively, Belcastro and Hull [18, 19] have proposed a model for rigid origami in which the deformation caused by folding is represented using affine transformations. This model provides constraints on the fold angles allowing for valid rigid origami configurations as well as mappings between unfolded and folded configurations. Such a model provided the theoretical basis for origami simulation tools such as the Rigid Origami Simulator [20] and Freeform Origami [21], both developed by Tachi (see Refs. [10, 22]).

Most origami modeling approaches and design tools to date are based on the assumption of creased folds (see Fig. 2(a)). These folds are straight line segments ${ }^{1}$ that divide the sheet into faces such that, upon folding, only zeroth-order geometric continuity $\left(G^{0}\right)$ is maintained between faces (i.e. the sheet tangent plane may be discontinuous at these folds). This idealization of physically folded structures has been useful in the analysis and design of many origami-inspired applications throughout the years [15, 26, 27]. However, such an idealization may not be appropriate for structures having non-negligible fold thickness or with maximum curvature at the folds

\footnotetext{
${ }^{1}$ Curved creased folds are also feasible in origami (information on curved creases is provided in Refs. [23, 24, 25]); however, the focus of this work is on rigid origami-based structures for which curved folds are not allowed since their folding induces bending of the faces connected to such folds [24].
}

restricted by material limitations (e.g. [28, 29, 30, 31]). In these cases, the folds are not accurately represented as creases but rather as bent sheet regions exhibiting higherorder geometric continuity (as shown in Figs. 1 and 2(b)). Such regions will be referred to in this work as smooth folds.

Modeling of bent and creased surfaces has been performed using collections of planar, cylindrical, conical, and other ruled surface subdomains [32, 33]. For example, Hwang and Yoon modeled three-dimensional developable surfaces via bending operations analogous to wrapping regions of an initially planar surface onto cylindrical and conical sections [33]. Zhu and coworkers developed a method for analyzing surfaces under creased and bent folds [34]. Their method allowed for the superposition of folds with arbitrary sharpness and fold angle that collectively dictated the ultimate shape of the analyzed surface. None of the aforementioned works $[32,33,34]$ has addressed constraints on the fold angles or fold pattern that are required to preserve rigidly deformed sheet regions as are formed in analogous rigid origami models, which are critical when fold intersections are present in the sheet.

Besides purely kinematic models, there have been efforts to model the structural response of origami sheets. Schenk and Guest [16] proposed a model for origami structures with elastic creased folds based on truss representations. Their model introduces torsional spring behavior at the creases. Tachi used a similar approach to model the elastic behavior of sheets with creased folds by also idealizing the folds as torsional springs and solving equations of mechanical equilibrium under constraints assuring that no fold line or boundary edge is elongated [35].

Representing folds as creases having torsional spring behavior may not be suitable for structures of significant thickness or comprised of materials that do not exhibit large enough strains to approximate creases (e.g. metal or active materials-based sheets). In these cases, the folds are properly modeled using plate or shell representations. A novel model for the structural (elastic) response of rigid origami structures having smooth folds is presented in this work. The higher-order continuity of smooth folds is required for their analysis using plate and shell representations. The presented kinematics provide constraints on the fold shape variables required for valid origami configurations. Such kinematic constraints are considered together with equations of mechanical equilibrium in order to model the elastic behavior origami sheets with smooth folds.

The outline of the paper is as follows: the theoretical preliminaries and the key kinematic equations for origami with smooth folds are presented in Sections 2 and 3, respectively. A model for origami sheets having elastic smooth folds is described in Section 4. A numerical approach used to implement this model is presented in Section 5. Details on the finite element and experimental analyses used to numerically and experimentally validate the proposed model are briefly presented in Sections 6 and 7 , respectively. Section 8 presents simulation results 
of origami sheets using the proposed model, and Section 9 provides a summarizing discussion and concludes the paper.

\section{Theoretical Preliminaries}

The origami modeling approach adopted and extended in this work is based on the model presented in Refs. [18, 19]. The studied continuum body denoted as the sheet and the shape variables associated with the smooth folds in the sheet (e.g. fold angles) are first described. The layout of the smooth folds (i.e. the fold pattern) is then determined by vertices (start-points and end-points of the line segments coincident with the smooth folds centerlines in a planar reference configuration) and their connectivity. Once the geometric parameters of the fold pattern are determined, constraints on the fold shape variables required for valid configurations are proposed. The set of configurations that satisfy the proposed constraints form the constrained configuration space [36, 37]. The continuous motion of the sheet represents a continuous path in the constrained configuration space. A more detailed description of the model for the kinematics of origami structures with smooth folds is presented in Ref. [9] and the main aspects are summarized here. Tables summarizing the nomenclature used in this work are provided in Appendix A.

The sheet is a three-dimensional, orientable, pathconnected surface with boundary. For origami with smooth folds, the sheet is divided into various surface subdomains denoted as the faces, the smooth folds, and the fold intersections. The orthonormal vectors $\mathbf{e}_{i} \in \mathbb{R}^{3}, i=$ $1,2,3$, with $\mathbf{e}_{3}:=\mathbf{e}_{1} \times \mathbf{e}_{2}$ form the basis $\left\{\mathbf{e}_{1}, \mathbf{e}_{2}, \mathbf{e}_{3}\right\}$ that defines the fixed global coordinate system.

The reference configuration of the sheet is denoted $\mathcal{S}_{0}$ and is defined such that it is contained in the plane spanned by $\mathbf{e}_{1}$ and $\mathbf{e}_{2}$ with its surface subdomains not overlapping each other, except at their shared boundary edges. The configuration of the $N_{\mathcal{P}}$ faces, $N_{\mathcal{F}}$ smooth folds, and $N_{\mathcal{I}}$ fold intersections in $\mathcal{S}_{0}$ are denoted $\mathcal{P}_{0}^{i}, i=1, \ldots, N_{\mathcal{P}}$, $\mathcal{F}_{0}^{i}, i=1, \ldots, N_{\mathcal{F}}$, and $\mathcal{I}_{0}^{i}, i=1, \ldots, N_{\mathcal{I}}$, respectively. Therefore, $\mathcal{S}_{0}=\left(\bigcup_{i=1}^{N_{\mathcal{P}}} \mathcal{P}_{0}^{i}\right) \cup\left(\bigcup_{i=1}^{N_{\mathcal{F}}} \mathcal{F}_{0}^{i}\right) \cup\left(\bigcup_{i=1}^{N_{\mathcal{I}}} \mathcal{I}_{0}^{i}\right)$. The side of $\mathcal{S}_{0}$ in its planar reference configuration with normal $\mathbf{e}_{3}$ is selected as the positive side of the sheet. An example of a sheet with smooth folds in its reference configuration $\mathcal{S}_{0}$ is shown in Fig. 3 .

The layout of the various surface subdomains comprising $\mathcal{S}_{0}$ is presented in a smooth fold pattern, defined here using the concept of graph thickening in topological graph theory [38]:

Definition 1. Smooth Fold Pattern: A smooth fold pattern on $\mathcal{S}_{0}$ is obtained by a $2 D$ thickening of a planar graph with edges embedded as straight line segments [38, 39]. Vertices on the boundary of $\mathcal{S}_{0}$ are not included in this thickening. Thus, $\mathcal{S}_{0}$ is formed by the union of $N_{\mathcal{F}}$ smooth folds $\mathcal{F}_{0}^{i}$ that correspond to the edges of the graph that thicken into rectangular strips, $N_{\mathcal{I}}$ fold intersections $\mathcal{I}_{0}^{i}$ that correspond to the vertices of the graph with valence $n_{i}$ that thicken into $2 n_{i}$-sided polygons, and the remainder of $\mathcal{S}_{0}$ is comprised by the $N_{\mathcal{P}}$ planar faces $\mathcal{P}_{0}^{i}$.

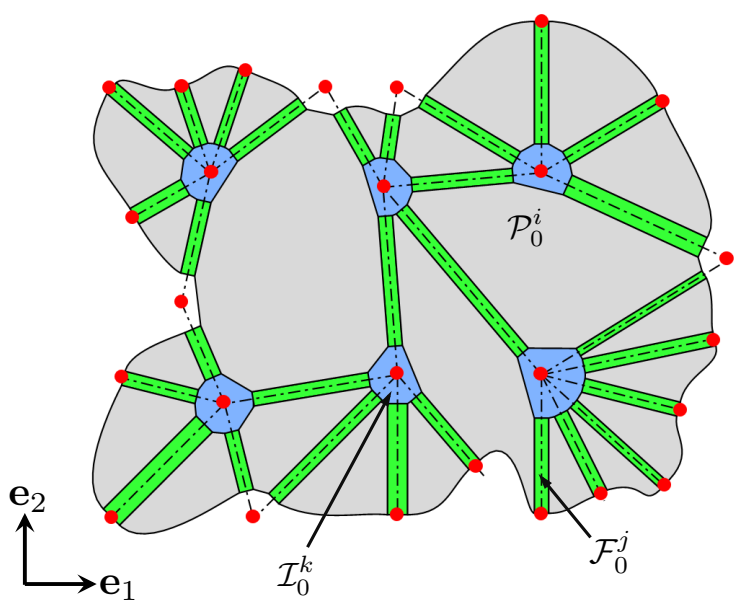

Reference configuration $\mathcal{S}_{0}$

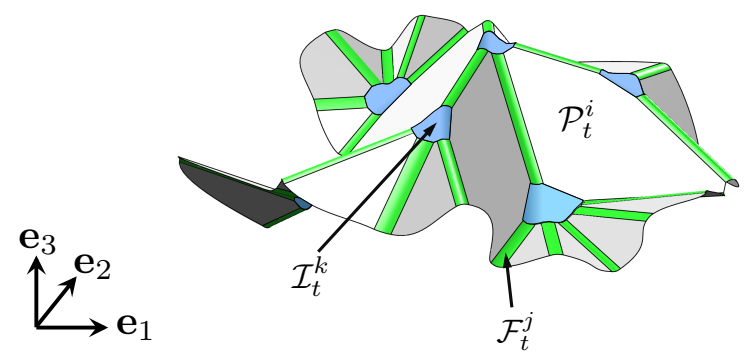

Current configuration $\mathcal{S}_{t}$

Figure 3: An origami sheet with smooth folds in its reference configuration $\mathcal{S}_{0}$ and a current configuration $\mathcal{S}_{t}$.

A current configuration of the sheet is denoted $\mathcal{S}_{t}$ where $t$ is a parameter that indicates the history of deformation from the reference configuration $(t=0)$ to a current configuration $(t>0)$. Refer to Fig. 3 for an example. The configuration of the $N_{\mathcal{P}}$ faces, $N_{\mathcal{F}}$ smooth folds, and $N_{\mathcal{I}}$ fold intersections comprising $\mathcal{S}_{t}$ are denoted $\mathcal{P}_{t}^{i}, i=1, \ldots, N_{\mathcal{P}}$, $\mathcal{F}_{t}^{i}, i=1, \ldots, N_{\mathcal{F}}$, and $\mathcal{I}_{t}^{i}, i=1, \ldots, N_{\mathcal{I}}$, respectively. Thus, $\mathcal{S}_{t}=\left(\bigcup_{i=1}^{N_{\mathcal{P}}} \mathcal{P}_{t}^{i}\right) \cup\left(\bigcup_{i=1}^{N_{\mathcal{F}}} \mathcal{F}_{t}^{i}\right) \cup\left(\bigcup_{i=1}^{N_{\mathcal{I}}} \mathcal{I}_{t}^{i}\right)$. As stated in Section 1 , the focus of this work is on rigid origami-based structures. As such, the following definition provides the characteristics of a valid current configuration for origami with smooth folds:

Definition 2. Valid configuration: $A$ valid current configuration $\mathcal{S}_{t}$ has the following characteristics: (i) The faces undergo only rigid deformations (i.e. they are neither bent nor stretched), (ii) the sheet is not torn (initially joined surface subdomains of the sheet remain joined), and (iii) the sheet does not self-intersect. 
Definition 3. Smooth folds: The smooth folds are ruled surfaces of the following form ${ }^{2}$ :

$$
\mathcal{F}_{t}^{i}\left(\zeta_{1}, \zeta_{2}\right)=\mathbf{c}_{t}^{i}\left(\zeta_{1}\right)+\zeta_{2} \mathbf{h}_{t}^{i}, \quad \frac{\mathrm{d} \mathbf{c}_{t}^{i}\left(\zeta_{1}\right)}{\mathrm{d} \zeta_{1}} \cdot \mathbf{h}_{t}^{i}=0
$$

where $\mathcal{F}_{t}^{i}\left(\zeta_{1}, \zeta_{2}\right) \in \mathbb{R}^{3}$ is a parameterization of $\mathcal{F}_{t}^{i}$. Without loss of generality, the parameters $\zeta_{1}$ and $\zeta_{2}$ are respectively contained in the intervals $[-1,1]$ and $[0,1]$.

In the previous definition, the vector $\mathbf{h}_{t}^{i} \in \mathbb{R}^{3}$ provides the direction of the rulings comprising $\mathcal{F}_{t}^{i}$ and $\mathbf{c}_{t}^{i}\left(\zeta_{1}\right) \in \mathbb{R}^{3}$ is the parametric curve that defines the cross-section of $\mathcal{F}_{t}^{i}$. The curve parameterized by $\mathbf{c}_{t}^{i}\left(\zeta_{1}\right)$ is contained in a plane orthogonal to $\mathbf{h}_{t}^{i}$ as stated in Eq. (1). It is assumed that $\left\|\mathbf{h}_{t}^{i}\right\|$ is constant ${ }^{3}$. As a consequence, the only non-rigid deformations allowed for the smooth folds are achieved through continuous deformation of their crosssection defined by $\mathbf{c}_{t}^{i}\left(\zeta_{1}\right)$ (i.e. bending or stretching of the fold cross-section). To simplify the notation, the dependence of $\mathcal{F}_{t}^{i}\left(\zeta_{1}, \zeta_{2}\right), \mathbf{c}_{t}^{i}\left(\zeta_{1}\right)$, and $\mathbf{h}_{t}^{i}$ on $t$ is left implicit for the remainder of the paper and these vectors are denoted as $\mathcal{F}^{i}\left(\zeta_{1}, \zeta_{2}\right), \mathbf{c}^{i}\left(\zeta_{1}\right)$, and $\mathbf{h}^{i}$, respectively. An example of a smooth fold is shown in Fig. 4.
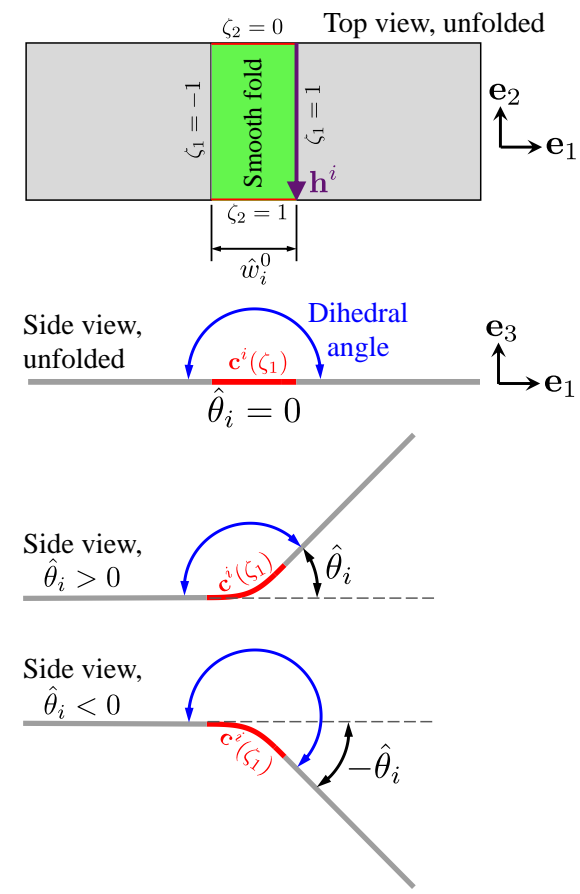

Figure 4: Schematics showing unfolded and folded configurations of a smooth fold.

Remark 1. Creased folds are a special limiting case of the more general smooth folds. Specifically, creased folds

\footnotetext{
${ }^{2} \mathbf{y}_{1} \cdot \mathbf{y}_{2}$ denotes the dot product of vectors $\mathbf{y}_{1}$ and $\mathbf{y}_{2}$.

$3\|\cdot\|$ denotes the 2 -norm, i.e. $\|\mathbf{y}\|=(\mathbf{y} \cdot \mathbf{y})^{1 / 2}$.
}

are obtained when the curve parameterized by $\mathbf{c}^{i}\left(\zeta_{1}\right)$ is degenerated to a single point thereby degenerating the smooth fold surface $\mathcal{F}_{t}^{i}$ to a single straight line segment.

Each smooth fold is joined to a face at each of its two boundary rulings (i.e. $\mathcal{F}^{i}\left(-1, \zeta_{2}\right)$ and $\mathcal{F}^{i}\left(1, \zeta_{2}\right)$, refer to Fig. 4). The remaining boundaries of $\mathcal{F}_{t}^{i}$ (i.e. $\mathcal{F}^{i}\left(\zeta_{1}, 0\right)$ and $\left.\mathcal{F}^{i}\left(\zeta_{1}, 1\right)\right)$ are either at the boundary of $\mathcal{S}_{t}$ or joined to a fold intersection. A parameterization for the fold intersections $\left(\mathcal{I}_{t}^{i}, i=1, \ldots, N_{\mathcal{I}}\right)$ is not provided here. It is noted, however, that the model proposed here is independent from the parameterization of $\mathcal{I}_{t}^{i}$.

A non-rigid body deformation of the sheet is achieved by rotating pairs of faces joined to smooth folds relative to one another in such a way that the sheet only attains valid configurations during this deformation. One of the shape variables associated with a smooth fold describes the relative rotation between the two faces joined by such a fold and is denoted as the fold angle:

Definition 4. Fold angle: The fold angle $\hat{\theta}_{i}(t)$ is defined as $\pi$ minus the dihedral angle between the positive sides of the two faces joined to the $i^{\text {th }}$ smooth fold.

The dependence of the fold angles on $t$ is left implicit for the remainder of the paper. Schematics showing the concept of fold angle for smooth folds are provided in Fig. 4. Let $\hat{\boldsymbol{\theta}} \in \mathbb{R}^{N_{\mathcal{F}}}$ be the vector constructed by collecting the fold angles as follows:

$$
\hat{\boldsymbol{\theta}}=\left[\begin{array}{lll}
\hat{\theta}_{1} & \ldots & \hat{\theta}_{N_{\mathcal{F}}}
\end{array}\right]^{\mathrm{T}} .
$$

Corollary 1. A current configuration $\mathcal{S}_{t}$ is valid if the deformed configuration of any closed path $\gamma(\eta):[0,1] \rightarrow$ $\mathcal{S}_{0}$ is also closed in $\mathcal{S}_{t}$.

This is a consequence of characteristic ( $i i)$ in Definition 2. For the sake of derivations, it is assumed that the paths $\gamma(\eta)$ do not cross any fold intersections.

Let the deformation associated with folding a smooth fold be represented as a matrix (denoted fold transformation matrix). The multiplication of the ordered fold transformation matrices associated with the folds crossed by a closed path $\gamma(\eta)$ results in the identity matrix $\mathbf{I}$ for a valid configuration. This implies that the mapping of positions is continuous and therefore no tearing occurs in the strip of faces and smooth folds crossed by $\gamma(\eta)$ (see Refs. [18, 19, 40] for details).

Lemma 1. Let $\mathbf{M}$ be the multiplication of fold transformation matrices associated with the folds crossed by an open path $\phi(\eta):[0,1] \rightarrow \mathcal{S}_{0}$ that does not cross any fold intersections. The multiplication of the fold transformation matrices associated with the folds crossed by $\phi^{-1}(\eta)$ (path $\phi(\eta)$ with reversed direction) is equal to $\mathbf{M}^{-1}$.

Proof. Let $\mathbf{M}_{*}$ be the multiplication of fold transformation matrices associated with the folds crossed by $\phi^{-1}(\eta)$. A closed path can be constructed by joining path $\phi(\eta)$ to $\phi^{-1}(\eta)$. Thus, $\mathbf{M M}_{*}=\mathbf{I}$ which implies $\mathbf{M}_{*}=\mathbf{M}^{-1}$. 
Lemma 2. In a valid current configuration, all paths in $\mathcal{S}_{0}$ that connect any two points on the planar faces yield the same multiplication of fold transformation matrices associated with the fold they cross. It is assumed that such paths do not cross any fold intersections.

Proof. Let $\phi_{1}(\eta):[0,1] \rightarrow \mathcal{S}_{0}, \phi_{2}(\eta):[0,1] \rightarrow \mathcal{S}_{0}$ be paths connecting any two points $\mathrm{A}$ and $\mathrm{B}$ in the faces of $\mathcal{S}_{0}$. Both paths do not cross any fold intersection. The multiplication of fold transformation matrices associated with each path are denoted $\mathbf{M}_{1}$ and $\mathbf{M}_{2}$, respectively. A closed path starting and ending at point A can be constructed by joining $\phi_{1}(\eta)$ to $\phi_{2}^{-1}(\eta)$. Thus, the multiplication of the fold transformation matrices of such a closed path must be the identity matrix: $\mathbf{M}_{1} \mathbf{M}_{2}^{-1}=\mathbf{I}$ which implies $\mathbf{M}_{1}=\mathbf{M}_{2}$.

Based on these foundations, a simplification that leads to a practical approach to describe valid configurations can be provided.

Definition 5. Vertex Module: A vertex module is a connected surface formed by union of a fold intersection $\mathcal{I}_{0}^{j}$ and its adjacent $n_{j}$ smooth folds and $n_{j}$ planar faces.

Figure 5 shows a smooth fold pattern with two interior fold intersections and their associated vertex modules.

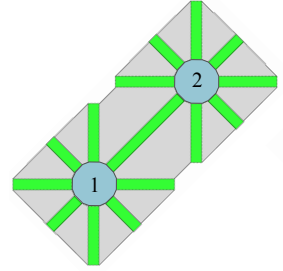

(a)

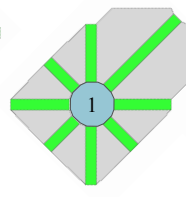

(b)

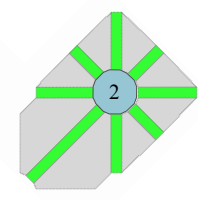

(c)
Figure 5: Example of a sheet with smooth folds having two vertex modules. (a) Smooth fold pattern. (b) and (c) The two vertex modules associated with this pattern.

Definition 6. Let $\gamma_{j}(\eta):[0,1] \rightarrow \mathcal{S}_{0}$ be a simple closed path in the vertex module associated with $\mathcal{I}_{0}^{j}$ that encloses $\mathcal{I}_{0}^{j}$. For simplicity it is assumed that $\boldsymbol{\gamma}_{j}(\eta)$ crosses each smooth fold in the vertex module only once.

Theorem 1. A current configuration is valid if the multiplication of fold transformation matrices associated with the folds crossed by $\gamma_{j}(\eta)$ is equal to $\mathbf{I}, \forall j \in\left\{1, \ldots, N_{\mathcal{I}}\right\} .{ }^{4}$

Proof. First consider two fold intersections, $\mathcal{I}_{0}^{k}$ and $\mathcal{I}_{0}^{l}$, that share a common adjacent smooth fold as shown in Fig. 6. The paths $\gamma_{k}(\eta)$ and $\gamma_{l}(\eta)$ are respectively contained in the vertex modules associated with $\mathcal{I}_{0}^{k}$ and $\mathcal{I}_{0}^{l}$.

\footnotetext{
${ }^{4}$ These conditions are necessary for a valid configuration. However, they are not sufficient since self-intersections are not taken into account in this work.
}

Then, consider a path $\gamma(\eta)$ that encloses both $\mathcal{I}_{0}^{k}$ and $\mathcal{I}_{0}^{l}$. The goal is to show that if the multiplication of fold transformation matrices associated with the folds crossed by $\gamma_{k}(\eta)$ and $\gamma_{l}(\eta)$ (denoted $\mathbf{M}_{k}$ and $\mathbf{M}_{l}$, respectively) are both equal to $\mathbf{I}$, the multiplication of fold transformations matrices for $\gamma(\eta)$ (denoted $\mathbf{M}$ ) is also equal to $\mathbf{I}$.

The matrix $\mathbf{M}_{k}$ can be expressed as $\mathbf{M}_{k}=\mathbf{M}_{k}^{*} \mathbf{L}=\mathbf{I}$ where $\mathbf{M}_{k}^{*}$ is the multiplication of fold transformation matrices associated with the folds crossed by $\gamma_{k}(\eta)$ except for the shared fold and $\mathbf{L}$ is the fold transformation matrix associated with the shared fold. The matrix $\mathbf{M}_{l}$ can be expressed as $\mathbf{M}_{l}=\mathbf{M}_{l}^{*} \mathbf{L}^{-1}=\mathbf{I}$ where $\mathbf{M}_{l}^{*}$ is the multiplication of fold transformation matrices associated with the folds crossed by $\gamma_{l}(\eta)$ excluding the fold transformation matrix of the shared fold. Note that the inverse of $\mathbf{L}$ appears in the expression for $\mathbf{M}_{l}$ since $\gamma_{l}(\eta)$ crosses the shared fold in opposite direction than $\gamma_{k}(\eta)$. Since $\gamma(\eta)$ crosses the same folds in the same direction as those crossed by $\gamma_{k}(\eta)$ followed by those crossed by $\gamma_{l}(\eta)$ except for their shared fold: $\mathbf{M}=\mathbf{M}_{k}^{*} \mathbf{M}_{l}^{*}=\mathbf{L}^{-1} \mathbf{L}=\mathbf{I}$. A similar approach can be utilized to show that every closed path in $\mathcal{S}_{0}$ that avoids fold intersections leads to a multiplication of fold transformation matrices equal to $\mathbf{I}$ provided that for each path $\gamma_{j}(\eta), j=1, \ldots, N_{\mathcal{I}}$, the multiplication of fold transformation matrices is equal to $\mathbf{I}$ (see Refs. [18, 19, 40]).

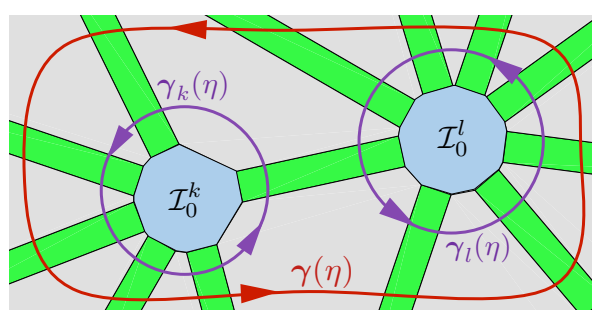

Figure 6: Paths $\gamma_{k}(\eta)$ and $\gamma_{l}(\eta)$ respectively enclosing the fold intersections $\mathcal{I}_{0}^{k}$ and $\mathcal{I}_{0}^{l}$ that share a common adjacent smooth fold. The path $\gamma(\eta)$ encloses both $\mathcal{I}_{0}^{k}$ and $\mathcal{I}_{0}^{l}$.

Theorem 1 allows for a simple mathematical description of valid configurations. Specifically, it allows to consider valid configuration constraints only for the paths $\gamma_{j}(\eta), j=1, \ldots, N_{\mathcal{I}}$, instead of all arbitrary paths in $\mathcal{S}_{0}$. Details on the geometry of the smooth folds, and explicit expressions for the fold transformation matrices and the kinematic constraints are provided in the subsequent section.

\section{Kinematics}

\subsection{Geometry of Smooth Folds}

The distance between the points with position vectors $\mathbf{c}^{i}(-1)$ and $\mathbf{c}^{i}(1)$ is denoted $\hat{w}_{i}$ :

$$
\hat{w}_{i}:=\left\|\mathbf{c}^{i}(1)-\mathbf{c}^{i}(-1)\right\| .
$$


A schematic of a smooth fold cross-section showing $\hat{w}_{i}$ is provided in Fig. 7. The fold width $\hat{w}_{i}^{0}$ is the value of $\hat{w}_{i}$ at the reference configuration $(t=0)$ (see Fig. 4).

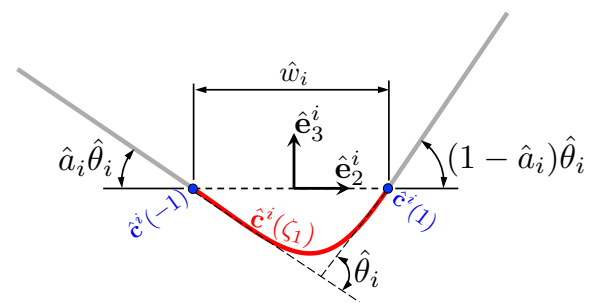

Figure 7: Cross-section of a smooth fold showing geometric variables and the fold-attached coordinate system.

The fold-attached orthonormal vectors $\hat{\mathbf{e}}_{j}^{i} \in \mathbb{R}^{3}, i=$ $1, \ldots, N_{\mathcal{F}}, j=1,2,3$, with $\hat{\mathbf{e}}_{3}^{i}:=\hat{\mathbf{e}}_{1}^{i} \times \hat{\mathbf{e}}_{2}^{i}$ form the bases $\left\{\hat{\mathbf{e}}_{1}^{i}, \hat{\mathbf{e}}_{2}^{i}, \hat{\mathbf{e}}_{3}^{i}\right\}$ that define the fold coordinate system of each smooth fold $\mathcal{F}_{t}^{i}$. The origin of this coordinate system is located at $\frac{1}{2} \mathbf{c}^{i}(-1)+\frac{1}{2} \mathbf{c}^{i}(1)$. The vector $\mathbf{h}^{i}$ is aligned to $\hat{\mathbf{e}}_{1}^{i}\left(\right.$ i.e. $\left.\mathbf{h}^{i} \cdot \hat{\mathbf{e}}_{1}^{i}=\left\|\mathbf{h}^{i}\right\|\right)$ and $\left(\mathbf{c}^{i}(1)-\mathbf{c}^{i}(-1)\right) \in \operatorname{span}\left(\hat{\mathbf{e}}_{2}^{i}\right)$.

The face adjacent to a smooth fold at the boundary $\mathcal{F}^{i}\left(-1, \zeta_{2}\right)$ makes an angle of $\hat{a}_{i} \hat{\theta}_{i}$ with $-\hat{\mathbf{e}}_{2}^{i}$ in the plane spanned by $\hat{\mathbf{e}}_{2}^{i}$ and $\hat{\mathbf{e}}_{3}^{i}$ and the face adjacent to a smooth fold at the boundary $\mathcal{F}^{i}\left(1, \zeta_{2}\right)$ makes an angle of $\left(1-\hat{a}_{i}\right) \hat{\theta}_{i}$ with $\hat{\mathbf{e}}_{2}^{i}$ in such a plane. This is shown schematically in Fig. 7. Let $\hat{\mathbf{c}}^{i}\left(\zeta_{1}\right)$ be the parametric curve $\mathbf{c}^{i}\left(\zeta_{1}\right)$ expressed in the fold coordinate system of $\mathcal{F}_{t}^{i}$ :

$$
\hat{\mathbf{c}}^{i}\left(\zeta_{1}\right)=\left[\begin{array}{lll}
\hat{\mathbf{e}}_{1}^{i} & \hat{\mathbf{e}}_{2}^{i} & \hat{\mathbf{e}}_{3}^{i}
\end{array}\right]^{\mathrm{T}}\left[\mathbf{c}^{i}\left(\zeta_{1}\right)-\frac{1}{2}\left(\mathbf{c}^{i}(-1)+\mathbf{c}^{i}(1)\right)\right] .
$$

The order of geometric continuity of $\mathcal{S}_{t}$ at the interior rulings of $\mathcal{F}_{t}^{i}$ is determined by the order of geometric continuity of $\hat{\mathbf{c}}^{i}\left(\zeta_{1}\right), \zeta_{1} \in(-1,1)$, while that at the joints with the planar faces adjacent to $\mathcal{F}_{t}^{i}$ depends on the particular values of $\hat{\mathbf{c}}_{i}\left(\zeta_{1}\right)$ and its derivatives at $\zeta_{1}= \pm 1$. For example, $G^{0}$ continuous joints with the faces adjacent to $\mathcal{F}_{t}^{i}$ require the following conditions on $\hat{\mathbf{c}}^{i}\left(\zeta_{1}\right)$ at $\zeta_{1}= \pm 1$ (refer to Fig. 7):

$$
\hat{\mathbf{c}}^{i}(-1)=\left[\begin{array}{c}
0 \\
-\frac{\hat{w}_{i}}{2} \\
0
\end{array}\right]=: \hat{\mathbf{c}}_{L_{0}}^{i}, \quad \hat{\mathbf{c}}^{i}(1)=\left[\begin{array}{c}
0 \\
\frac{\hat{w}_{i}}{2} \\
0
\end{array}\right]=: \hat{\mathbf{c}}_{R_{0}}^{i}
$$

Continuity of the unit tangent vector of $\hat{\mathbf{c}}^{i}\left(\zeta_{1}\right)$ at $\zeta_{1}=$ \pm 1 is required for $G^{1}$ continuous joints with the planar faces adjacent to $\mathcal{F}_{t}^{i}$ in addition to $G^{0}$ continuity $[41,42]$. Therefore, in addition to those conditions of Eq. (5), the following conditions on the first derivatives of $\hat{\mathbf{c}}^{i}\left(\zeta_{1}\right)$ at $\zeta_{1}= \pm 1$ are required for $G^{1}$ continuity at the joints with the planar faces adjacent to $\mathcal{F}_{t}^{i}$ :

$$
\begin{gathered}
\left.\frac{\mathrm{d} \hat{\mathbf{c}}^{i}\left(\zeta_{1}\right)}{\mathrm{d} \zeta_{1}}\right|_{\zeta_{1}=-1}=\beta_{L_{1}}^{i}\left[\begin{array}{c}
0 \\
\cos \left(\hat{a}_{i} \hat{\theta}_{i}\right) \\
-\sin \left(\hat{a}_{i} \hat{\theta}_{i}\right)
\end{array}\right]=: \hat{\mathbf{c}}_{L_{1}}^{i}, \\
\left.\frac{\mathrm{d} \hat{\mathbf{c}}^{i}\left(\zeta_{1}\right)}{\mathrm{d} \zeta_{1}}\right|_{\zeta_{1}=1}=\beta_{R_{1}}^{i}\left[\begin{array}{c}
0 \\
\cos \left(\left(1-\hat{a}_{i}\right) \hat{\theta}_{i}\right) \\
\sin \left(\left(1-\hat{a}_{i}\right) \hat{\theta}_{i}\right)
\end{array}\right]=: \hat{\mathbf{c}}_{R_{1}}^{i},
\end{gathered}
$$

where $\beta_{L_{1}}^{i}, \beta_{R_{1}}^{i} \in \mathbb{R}_{>0}$. Conditions for higher-order geometric continuity can be derived in a similar manner [9].

Polynomials of the minimum order required to satisfy the previous conditions for continuity of $\hat{\mathbf{c}}^{i}\left(\zeta_{1}\right)$ and its derivatives at $\zeta_{1}= \pm 1$ are used to define this parametric curve. Hermitian interpolation polynomials [43] are used to represent $\hat{\mathbf{c}}^{i}\left(\zeta_{1}\right)$ in this work. For $G^{1}$ continuity at the boundary rulings of $\mathcal{F}_{t}^{i}, \hat{\mathbf{c}}^{i}\left(\zeta_{1}\right)$ is expressed as follows:

$\hat{\mathbf{c}}^{i}\left(\zeta_{1}\right)=h_{30}\left(\zeta_{1}\right) \hat{\mathbf{c}}_{L_{0}}^{i}+h_{31}\left(\zeta_{1}\right) \hat{\mathbf{c}}_{R_{0}}^{i}+h_{32}\left(\zeta_{1}\right) \hat{\mathbf{c}}_{L_{1}}^{i}+h_{33}\left(\zeta_{1}\right) \hat{\mathbf{c}}_{R_{1}}^{i}$,

where the utilized cubic Hermitian interpolation polynomials $h_{3 i}, i=0, \ldots, 3$, are given as follows:

$$
\begin{aligned}
& h_{30}\left(\zeta_{1}\right)=\frac{1}{4} \zeta_{1}^{3}-\frac{3}{4} \zeta_{1}+\frac{1}{2}, \quad h_{31}\left(\zeta_{1}\right)=-\frac{1}{4} \zeta_{1}^{3}+\frac{3}{4} \zeta_{1}+\frac{1}{2} \\
& h_{32}\left(\zeta_{1}\right)=\frac{1}{4} \zeta_{1}^{3}-\frac{1}{4} \zeta_{1}^{2}-\frac{1}{4} \zeta_{1}+\frac{1}{4} \\
& h_{33}\left(\zeta_{1}\right)=\frac{1}{4} \zeta_{1}^{3}+\frac{1}{4} \zeta_{1}^{2}-\frac{1}{4} \zeta_{1}-\frac{1}{4} .
\end{aligned}
$$

\subsection{Smooth Fold Pattern}

To describe the smooth fold pattern (i.e. the layout of the smooth folds in $\mathcal{S}_{0}$ ), the vertices are first introduced:

Definition 7. Vertices: The vertices are the start-points and the end-points of the line segments coincident with the centerlines of the smooth folds in $\mathcal{S}_{0}$. Each vertex has an associated position vector denoted $\mathbf{v}^{j} \in \operatorname{span}\left(\mathbf{e}_{1}, \mathbf{e}_{2}\right)$.

The number of vertices located at the interior of $\mathcal{S}_{0}$ is denoted $N_{\mathcal{I}}$ and the number of vertices located at the boundary of $\mathcal{S}_{0}$ or outside $\mathcal{S}_{0}$ is denoted $N_{\mathcal{B}}$. The vertices are enumerated starting from those located at the interior of $\mathcal{S}_{0}$ (with corresponding position vectors $\mathbf{v}^{1}, \ldots, \mathbf{v}^{N_{\mathcal{I}}}$ ) followed by those located at the boundary of $\mathcal{S}_{0}$ or outside $\mathcal{S}_{0}$ (with corresponding position vectors $\mathbf{v}^{N_{\mathcal{I}}+1}, \ldots, \mathbf{v}^{N_{\mathcal{I}}+N_{\mathcal{B}}}$ ).

Let $\hat{\mathbf{v}}^{i 1}, \hat{\mathbf{v}}^{i 2} \in \operatorname{span}\left(\mathbf{e}_{1}, \mathbf{e}_{2}\right), i=1, \ldots, N_{\mathcal{F}}$, be the position vectors of the vertices from which each fold centerline emanates and ends, respectively. These positions vectors are obtained from the sheet vertex position vectors $\mathbf{v}^{1}, \ldots, \mathbf{v}^{N_{\mathcal{I}}+N_{\mathcal{B}}}$ through a connectivity matrix (see Ref. [9] for details). 
The four corner points of $\mathcal{F}_{0}^{i}$ having associated position vectors $\hat{\mathbf{p}}_{1}^{i}, \hat{\mathbf{p}}_{2}^{i}, \hat{\mathbf{p}}_{3}^{i}, \hat{\mathbf{p}}_{4}^{i} \in \operatorname{span}\left(\mathbf{e}_{1}, \mathbf{e}_{2}\right), i=1, \ldots, N_{\mathcal{F}}$, are then determined as shown in Fig. 8 where $\hat{r}_{1}^{i}, \hat{r}_{2}^{i} \in \mathbb{R}$ and the resulting fold length orthogonal to the width direction must be positive (i.e. $\left\|\hat{\mathbf{v}}^{i 2}-\hat{\mathbf{v}}^{i 1}\right\|-\hat{r}_{1}^{i}-\hat{r}_{2}^{i}>0$ ).
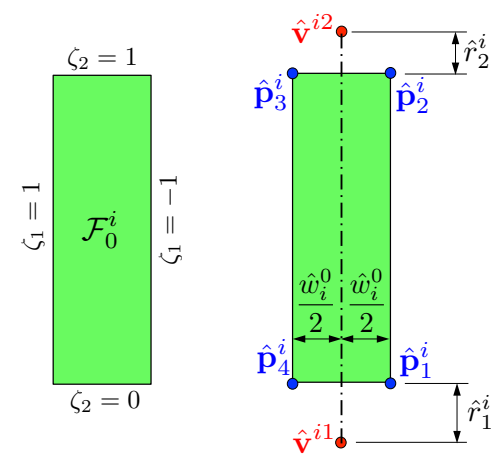

Figure 8: Geometric parameters defining $\mathcal{F}_{0}^{i}$.

Let $n_{j}, j=1, \ldots, N_{\mathcal{I}}$, be the number of fold centerlines incident to each interior vertex (i.e. those vertices located at the interior of $\mathcal{S}_{0}$ ).

Definition 8. The angle between the vector $\mathbf{y} \in$ $\operatorname{span}\left(\mathbf{e}_{1}, \mathbf{e}_{2}\right)$ and $\mathbf{e}_{1}$, starting at $\mathbf{e}_{1}$ and measured in the counterclockwise direction, is denoted $\varphi(\mathbf{y})$ : $\operatorname{span}\left(\mathbf{e}_{1}, \mathbf{e}_{2}\right) \rightarrow[0,2 \pi)$ and is determined as follows:

$$
\varphi(\mathbf{y}):=\left\{\begin{array}{rl}
\cos ^{-1}\left(\frac{\mathbf{y}}{\|\mathbf{y}\|} \cdot \mathbf{e}_{1}\right) ; & \mathbf{e}_{2} \cdot \mathbf{y} \geq 0 \\
2 \pi-\cos ^{-1}\left(\frac{\mathbf{y}}{\|\mathbf{y}\|} \cdot \mathbf{e}_{1}\right) ; & \mathbf{e}_{2} \cdot \mathbf{y}<0
\end{array} .\right.
$$

Let $\mathbf{m}^{j k} \in \operatorname{span}\left(\mathbf{e}_{1}, \mathbf{e}_{2}\right), j=1, \ldots, N_{\mathcal{I}}, k=1, \ldots, n_{j}$, be the vector along the length of the $k^{\text {th }}$ fold centerline incident to the $j^{\text {th }}$ interior vertex that emanates from this vertex (see Fig. 9). The vectors $\mathbf{m}^{j k}, k=1, \ldots, n_{j}$, have a counterclockwise ordering, i.e. $\varphi\left(\mathbf{m}^{j 1}\right)<\varphi\left(\mathbf{m}^{j 1}\right)<\cdots<$ $\varphi\left(\mathbf{m}^{j n_{j}}\right) \quad \forall j \in\left\{1, \ldots, N_{\mathcal{I}}\right\}$.

The angles between adjacent fold centerlines intersecting at a common interior vertex are denoted $\alpha_{j k}, j=$ $1, \ldots, N_{\mathcal{I}}, k=1, \ldots, n_{j}$. A schematic showing the vectors $\mathbf{m}^{j k}$ and the angles $\alpha_{j k}$ is provided in Fig. 9.

\subsection{Constraints}

As in conventional origami with creased folds [18, 19], constraints are required for origami with smooth folds to ensure that every current configuration $\mathcal{S}_{t}$ is valid ${ }^{5}$ (according to Definition 2).

\footnotetext{
${ }^{5}$ As stated in Section 2, self-intersection avoidance is not considered in this work.
}

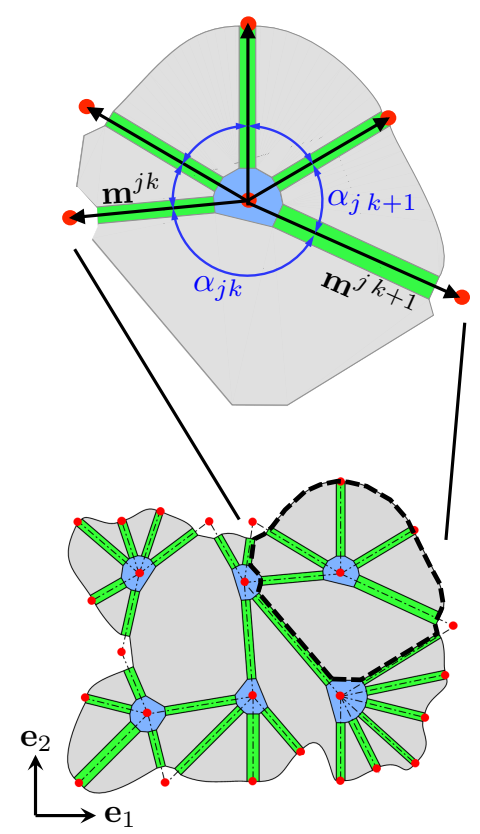

Figure 9: An interior vertex module and its associated geometric parameters.

The variables describing the deformation associated with the folding of the smooth folds are constrained such that every configuration $\mathcal{S}_{t}$ is valid. These variables correspond to the fold angle $\hat{\theta}_{i}$, the distance $\hat{w}_{i}$ between the endpoints of the cross-section parametric curve $\hat{\mathbf{c}}^{i}\left(\zeta_{1}\right)$, and $\hat{a}_{i}$, $i=1, \ldots, N_{\mathcal{F}}$ (refer to Section 3.1). As a consequence of Theorem 1, a set of constraints for the variables associated with the smooth folds adjacent to each interior fold intersection is proposed (i.e. the ordered folds crossed by the paths $\gamma_{j}(\eta), j=1, \ldots, N_{\mathcal{I}}$, see Definition 6$)$.

The variables $\theta_{j k}, w_{j k}$, and $a_{j k}$ are those associated with the $k^{\text {th }}$ smooth fold adjacent to $\mathcal{I}_{0}^{j}$. These variables are obtained from $\hat{\theta}_{i}, \hat{w}_{i}$, and $\hat{a}_{i}, i=1, \ldots, N_{\mathcal{F}}$, via a connectivity matrix (see Ref. [9] for details). Recalling Definition $6, \gamma_{j}(\eta)$ is a simple closed path enclosing $\mathcal{I}_{0}^{j}$ and that crosses each smooth fold adjacent to $\mathcal{I}_{0}^{j}$ once. An example of a path $\gamma_{j}(\eta)$ is shown in Fig. 10. The point having position $\gamma_{j}(0)$ is defined such that it is located at the face adjacent to the smooth folds with corresponding vectors $\mathbf{m}^{j 1}$ and $\mathbf{m}^{j n_{j}}$. Also, the path $\gamma_{j}(\eta)$ is defined such that it crosses the smooth folds with associated vectors $\mathbf{m}^{j k}$ in counterclockwise order (i.e. $\mathbf{m}^{j 1}, \mathbf{m}^{j 2}, \ldots, \mathbf{m}^{j n_{j}}$ ). The position vectors of the points where $\gamma_{j}(\eta)$ crosses each boundary ruling of the smooth folds are denoted $\mathbf{b}_{L}^{j k} \in \operatorname{span}\left(\mathbf{e}_{1}, \mathbf{e}_{2}\right)$ (point where $\gamma_{j}(\eta)$ enters the smooth fold) and $\mathbf{b}_{R}^{j k} \in \operatorname{span}\left(\mathbf{e}_{1}, \mathbf{e}_{2}\right)$ (point where $\gamma_{j}(\eta)$ exits the smooth fold). This is shown schematically in Fig. 10(a).

Let $\mathbf{Q}_{1}(\phi) \in \mathbb{R}^{4 \times 4}$ be the transformation matrix in homogeneous coordinates associated with a rotation by $\phi$ about an axis aligned to $\mathbf{e}_{1}$ : 


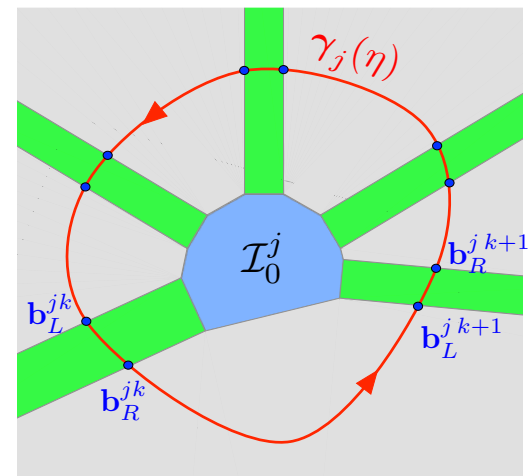

(a)

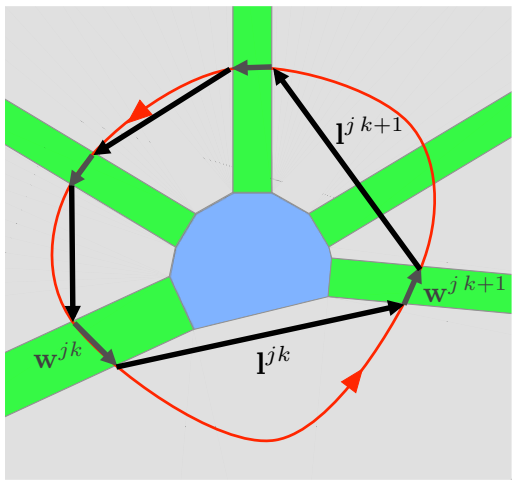

(b)

Figure 10: (a) Path $\gamma_{j}(\eta)$ crossing the faces and smooth folds joined to $\mathcal{I}_{0}^{j}$. (b) Vectors $\mathbf{w}^{j k}$ and $\mathbf{l}^{j k}$ with start-points and end-points corresponding to the points where the path $\gamma_{j}(\eta)$ crosses the boundary rulings of the smooth folds.

$\mathbf{Q}_{1}(\phi)=\left[\begin{array}{cccc}1 & 0 & 0 & 0 \\ 0 & \cos (\phi) & -\sin (\phi) & 0 \\ 0 & \sin (\phi) & \cos (\phi) & 0 \\ 0 & 0 & 0 & 1\end{array}\right]=\left[\begin{array}{cc}\mathbf{R}_{1}(\phi) & \mathbf{0}_{3} \\ \mathbf{0}_{3}^{\mathrm{T}} & 1\end{array}\right]$,

where $\mathbf{0}_{n}$ is the zero vector in $\mathbb{R}^{n}$. Let $\mathbf{Q}_{3}(\phi) \in \mathbb{R}^{4 \times 4}$ be the transformation matrix in homogeneous coordinates associated with a rotation by $\phi$ about an axis aligned to $\mathbf{e}_{3}$ :

$\mathbf{Q}_{3}(\phi)=\left[\begin{array}{cccc}\cos (\phi) & -\sin (\phi) & 0 & 0 \\ \sin (\phi) & \cos (\phi) & 0 & 0 \\ 0 & 0 & 1 & 0 \\ 0 & 0 & 0 & 1\end{array}\right]=\left[\begin{array}{cc}\mathbf{R}_{3}(\phi) & \mathbf{0}_{3} \\ \mathbf{0}_{3}^{\mathrm{T}} & 1\end{array}\right]$

Also, let $\mathbf{T}(\mathbf{b}) \in \mathbb{R}^{4 \times 4}$ be the matrix representing the transformation in homogeneous coordinates associated with a translation by vector $\mathbf{b} \in \mathbb{R}^{3}$ :

$$
\mathbf{T}(\mathbf{b})=\left[\begin{array}{cccc}
1 & 0 & 0 & b_{1} \\
0 & 1 & 0 & b_{2} \\
0 & 0 & 1 & b_{3} \\
0 & 0 & 0 & 1
\end{array}\right]=\left[\begin{array}{cc}
\mathbf{I}_{3} & \mathbf{b} \\
\mathbf{0}_{3}^{\mathrm{T}} & 1
\end{array}\right],
$$

where $\mathbf{I}_{n}$ is the $\mathbb{R}^{n \times n}$ identity matrix. Considering an axis with direction aligned to a vector $\mathbf{y} \in \operatorname{span}\left(\mathbf{e}_{1}, \mathbf{e}_{2}\right)$ that crosses a point with position vector $\mathbf{b} \in \operatorname{span}\left(\mathbf{e}_{1}, \mathbf{e}_{2}\right)$, the transformation associated with a rotation by $\phi$ about such an axis is as follows $[18,19]$ :

$$
\mathbf{T}(\mathbf{b}) \mathbf{Q}_{3}(\varphi(\mathbf{y})) \mathbf{Q}_{1}(\phi) \mathbf{Q}_{3}^{-1}(\varphi(\mathbf{y})) \mathbf{T}^{-1}(\mathbf{b}) .
$$

Theorem 2. The fold transformation matrix $\mathbf{L}^{j k}$ that describes the deformation associated with the folding of the $k^{\text {th }}$ smooth fold crossed by the path $\gamma_{j}(\eta)$ has the following form:

$$
\begin{gathered}
\mathbf{L}^{j k}=\left(\mathbf{T}\left(\mathbf{b}_{L}^{j k}-\mathbf{g}^{j k-1}\right) \mathbf{Q}_{3}\left(\varphi\left(\mathbf{m}^{j k}\right)\right) \mathbf{Q}_{1}\left(a_{j k} \theta_{j k}\right)\right. \\
\mathbf{Q}_{3}^{-1}\left(\varphi\left(\mathbf{m}^{j k}\right)\right) \mathbf{T}^{-1}\left(\mathbf{b}_{L}^{j k}-\mathbf{g}^{j k-1}\right) \\
\mathbf{T}\left(\mathbf{b}_{R}^{j k}-\mathbf{g}^{j k}\right) \mathbf{Q}_{3}\left(\varphi\left(\mathbf{m}^{j k}\right)\right) \mathbf{Q}_{1}\left(\left(1-a_{j k}\right) \theta_{j k}\right) \\
\left.\mathbf{Q}_{3}^{-1}\left(\varphi\left(\mathbf{m}^{j k}\right)\right) \mathbf{T}^{-1}\left(\mathbf{b}_{R}^{j k}-\mathbf{g}^{j k}\right)\right)
\end{gathered}
$$

where the vectors $\mathbf{g}^{j k} \in \operatorname{span}\left(\mathbf{e}_{1}, \mathbf{e}_{2}\right), k=0, \ldots, n_{j}$, account for the change in the distance between the boundary rulings of the smooth folds in a current configuration and are determined recursively as follows:

$$
\begin{aligned}
\mathbf{g}^{j 0}= & \mathbf{0}_{3}, \\
\mathbf{g}^{j k}= & \mathbf{g}^{j k-1} \\
& \quad+\left(w_{j k}^{0}-w_{j k}\right)\left(\mathbf{e}_{3} \times \frac{\mathbf{m}^{j k}}{\left\|\mathbf{m}^{j k}\right\|}\right) ; \quad k>0 .
\end{aligned}
$$

Proof. Considering the schematics shown in Figs. 11(c) and 11(d), the deformation associated with the folding of the $k^{\text {th }}$ smooth fold crossed by $\gamma_{j}(\eta)$ can be discretized by two consecutive rotation transformations. The first transformation corresponds to a rotation by $\left(1-a_{j k}\right) \theta_{j k}$ about an axis aligned to $\mathbf{m}^{j k}$ and crossing a point with position vector $\mathbf{b}_{R}^{j k}-\mathbf{g}^{j k}$. Using Eq. (14), the transformation matrix associated with this rotation is the following:

$$
\begin{gathered}
\left(\mathbf{T}\left(\mathbf{b}_{R}^{j k}-\mathbf{g}^{j k}\right) \mathbf{Q}_{3}\left(\varphi\left(\mathbf{m}^{j k}\right)\right) \mathbf{Q}_{1}\left(\left(1-a_{j k}\right) \theta_{j k}\right)\right. \\
\left.\left.\mathbf{Q}_{3}^{-1}\left(\varphi\left(\mathbf{m}^{j k}\right)\right) \mathbf{T}^{-1}\left(\mathbf{b}_{R}^{j k}-\mathbf{g}^{j k}\right)\right)\right) .
\end{gathered}
$$

The second transformation corresponds to a rotation by $a_{j k} \theta_{j k}$ about an axis aligned to $\mathbf{m}^{j k}$ and a crossing point 
with position vector $\mathbf{b}_{L}^{j k}-\mathbf{g}^{j k-1}$. The transformation matrix associated with this rotation is the following:

$$
\begin{gathered}
\left(\mathbf{T}\left(\mathbf{b}_{L}^{j k}-\mathbf{g}^{j k-1}\right) \mathbf{Q}_{3}\left(\varphi\left(\mathbf{m}^{j k}\right)\right) \mathbf{Q}_{1}\left(a_{j k} \theta_{j k}\right)\right. \\
\left.\mathbf{Q}_{3}^{-1}\left(\varphi\left(\mathbf{m}^{j k}\right)\right) \mathbf{T}^{-1}\left(\mathbf{b}_{L}^{j k}-\mathbf{g}^{j k-1}\right)\right) .
\end{gathered}
$$

The multiplication of the transformations shown in Eqs. (17) and (18) results in the transformation matrix $\mathbf{L}^{j k}$ provided in Eq. (15).

Referring to Figs. 11(a) and 11(b), the change in the distance between the boundary rulings of a smooth fold in a current configuration is $-\left(w_{j k}^{0}-w_{j k}\right)$. To define the position of the axes of rotation for the transformations composing $\mathbf{L}^{j k}$, the vector $\sum_{l=1}^{k-1}\left(w_{j l}^{0}-w_{j l}\right)\left(\mathbf{e}_{3} \times \frac{\mathbf{m}^{j l}}{\left\|\mathbf{m}^{j l}\right\|}\right)$ must be subtracted from $\mathbf{b}_{L}^{j k}$ and the vector $\sum_{l=1}^{k}\left(w_{j l}^{0}-\right.$ $\left.w_{j l}\right)\left(\mathbf{e}_{3} \times \frac{\mathbf{m}^{j l}}{\left\|\mathbf{m}^{j l}\right\|}\right)$ must be subtracted from $\mathbf{b}_{R}^{j k}$. The recursive definition of the vectors $\mathbf{g}^{j k}, k=0, \ldots, n_{j}$, in Eq. (16) allows for a simplified form of these vector subtractions.

Let the vectors $\mathbf{w}^{j k}, \mathbf{l}^{j k} \in \operatorname{span}\left(\mathbf{e}_{1}, \mathbf{e}_{2}\right)$ be defined as follows:

$$
\begin{gathered}
\mathbf{w}^{j k}:=\mathbf{b}_{R}^{j k}-\mathbf{g}^{j k}-\mathbf{b}_{L}^{j k}+\mathbf{g}^{j k-1}, \\
\mathbf{l}^{j k}:=\left\{\begin{array}{cl}
\mathbf{b}_{L}^{j k+1}-\mathbf{b}_{R}^{j k} ; & k=1, \ldots, n_{j}-1 \\
\mathbf{b}_{L}^{j 1}-\mathbf{b}_{R}^{j k} ; & k=n_{j}
\end{array} .\right.
\end{gathered}
$$

The vectors $\mathbf{w}^{j k}$ and $\mathbf{l}^{j k}$ for the example in Fig. 10(a) are shown in Fig. 10(b). Let the vectors $\tilde{\mathbf{w}}^{j k}$ and $\tilde{\mathbf{l}}^{j k}$ be $\mathbf{w}^{j k}$ and $\mathbf{l}^{j k}$, respectively, expressed in a coordinate system with the 1 -axis aligned to $\mathbf{m}^{j k}$ and the 3 -axis aligned to $\mathbf{e}_{3}$ :

$$
\begin{aligned}
\tilde{\mathbf{w}}^{j k} & =\mathbf{R}_{3}^{-1}\left(\varphi\left(\mathbf{m}^{j k}\right)\right) \mathbf{w}^{j k}, \\
\tilde{\mathbf{l}}^{j k} & =\mathbf{R}_{3}^{-1}\left(\varphi\left(\mathbf{m}^{j k}\right)\right) \mathbf{l}^{j k}
\end{aligned}
$$

Following the process presented in Refs. [18, 19], to formulate constraints for the shape variables associated with the folds, the mapping between reference and current configurations considering only the faces and smooth folds adjacent to an interior fold intersection is first constructed. As such, the face containing the point with position $\gamma_{j}(0)$ is assumed fixed for the derivation of constraints. Let $\mathbf{X} \in \operatorname{span}\left(\mathbf{e}_{1}, \mathbf{e}_{2}\right)$ be the position vector of a point in a face joined to $\mathcal{I}_{0}^{j}$ in the reference configuration. Also, let $\mathbf{x} \in \mathbb{R}^{3}$ be the position vector of such a point in a current configuration. The mapping $\mathbf{X} \mapsto \mathbf{x}$ is constructed as the multiplication of transformations $\mathbf{L}^{j k}$ associated with the folds crossed by the segment of path $\gamma_{j}(\eta)$ that connects $\gamma_{j}(0)$ to the face containing the point with initial position $\mathrm{X}$ :
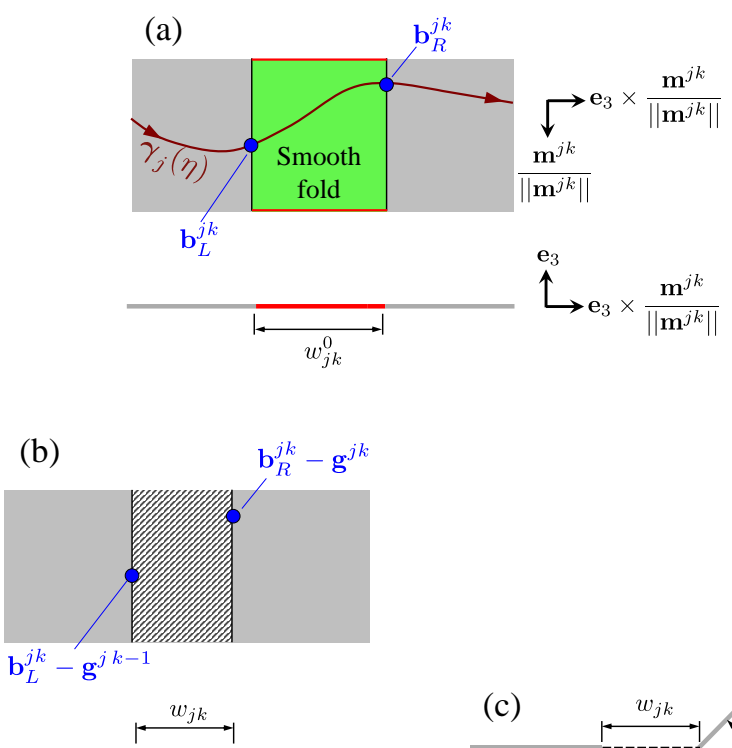

(c)

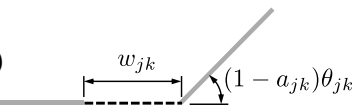

(d)

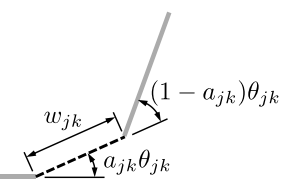

(e)

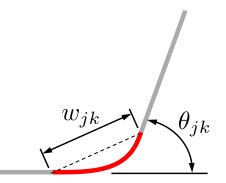

Figure 11: Schematics illustrating the process for the derivation of the transformation matrix associated with the folding of the $k^{\text {th }}$ smooth fold crossed by $\gamma_{j}(\eta)$. (a) Fold in its reference configuration. (b) Intermediate step to determine the location of the axes of rotation taking into account the change in the distance between the boundary rulings of the smooth fold in a current configuration. The vector $\mathbf{g}^{j k-1}$ is subtracted from $\mathbf{b}_{L}^{j k}$ to account for the previous smooth folds crossed by $\gamma_{j}(\eta)$. (c) Rotation by $\left(1-a_{j k}\right) \theta_{j k}$ about an axis aligned to $\mathbf{m}^{j k}$ and crossing a point with position vector $\mathbf{b}_{R}^{j k}-\mathbf{g}^{j k}$. (d) Rotation by $a_{j k} \theta_{j k}$ about an axis aligned to $\mathbf{m}^{j k}$ and a crossing point with position vector $\mathbf{b}_{L}^{j k}-\mathbf{g}^{j k-1}$. (e) Resulting configuration of the smooth fold and its adjacent faces.

$$
\left[\begin{array}{c}
\mathbf{x} \\
1
\end{array}\right]=\left(\prod_{k=1}^{n_{y}} \mathbf{L}^{j k}\right) \mathbf{T}^{-1}\left(\mathbf{g}^{j n_{y}}\right)\left[\begin{array}{c}
\mathbf{X} \\
1
\end{array}\right]
$$

where $n_{y}$ is the number of smooth folds crossed by the segment of the path $\gamma_{j}(\eta)$ that connects $\gamma_{j}(0)$ and the face containing the point with position vector $\mathbf{X}$. Since this mapping is a multiplication of translation and rotation matrices, each face undergoes a rigid deformation (required for a valid configuration). In order to prevent tearing among the surface subdomains joined to $\mathcal{I}_{t}^{j}$, the following constraints are proposed:

Theorem 3. For the initially closed strip of faces and smooth folds joined to $\mathcal{I}_{0}^{j}$ to remain closed with each face undergoing a rigid deformation, the following constraints must hold: 


$$
\mathbf{R}^{j}:=\prod_{k=1}^{n_{j}} \mathbf{R}_{1}\left(\theta_{j k}\right) \mathbf{R}_{3}\left(\alpha_{j k}\right)=\mathbf{I}_{3}
$$

and:

$$
\begin{aligned}
\mathbf{d}^{j}:=\sum_{k=1}^{n_{j}} & \left(\left(\prod_{l=1}^{k-1} \mathbf{R}_{1}\left(\theta_{j l}\right) \mathbf{R}_{3}\left(\alpha_{j l}\right)\right) \mathbf{R}_{1}\left(a_{j k} \theta_{j k}\right) \tilde{\mathbf{w}}^{j k}\right. \\
& \left.+\left(\prod_{l=1}^{k-1} \mathbf{R}_{1}\left(\theta_{j l}\right) \mathbf{R}_{3}\left(\alpha_{j l}\right)\right) \mathbf{R}_{1}\left(\theta_{j k}\right) \tilde{\mathbf{l}}^{j k}\right)=\mathbf{0}_{3} .
\end{aligned}
$$

Proof. Considering Theorem 1, the multiplication of all the fold transformation matrices associated with the folds crossed by $\gamma_{j}(\eta)$ must yield the identity transformation (analogous to previous work in origami with creased folds [18]):

$$
\left(\prod_{k=1}^{n_{j}} \mathbf{L}^{j k}\right) \mathbf{T}^{-1}\left(\mathbf{g}^{j n_{j}}\right)=\mathbf{I}_{4} \text {. }
$$

It can be shown after some matrix algebra steps ${ }^{6}$ that the 11 block of the left side of the matrix equation (26) is equal to $\mathbf{I}_{3}$ if $\mathbf{R}^{j}$ defined in Eq. (24) is equal to $\mathbf{I}_{3}$. Likewise, it can be shown that the 12 block of the left side of the matrix equation (26) is equal to $\mathbf{0}_{3}$ if $\mathbf{d}^{j}$ defined in Eq. (25) is equal to $\mathbf{0}_{3}$. The 21 and 22 blocks are equal to $\mathbf{0}_{3}^{\mathrm{T}}$ and 1 , respectively, independently of the fold shape variables.

The consideration of both Theorems 1 and 3 yields the following Corollary that provides necessary conditions, in the form of explicit kinematic constraints, for the fold shape variables allowing for a valid configuration (excluding self-intersection avoidance):

Corollary 2. The constraints on the fold shape variables allowing for a valid configuration (excluding selfintersection avoidance) for a sheet with smooth folds are the following:

$$
\mathbf{R}^{j}=\mathbf{I}_{3}, \quad \mathbf{d}^{j}=\mathbf{0}_{3} \quad \forall j \in\left\{1, \ldots, N_{\mathcal{I}}\right\} .
$$

In general, $\hat{\theta}_{i}, \hat{w}_{i}$ and $\hat{a}_{i}, i=1, \ldots, N_{\mathcal{F}}$, represent the shape variables (i.e. degrees of freedom) that describe the deformation of the sheet. If certain assumptions are taken for the deformation of each individual smooth fold, its associated variables $\hat{\theta}_{i}, \hat{w}_{i}$, and $\hat{a}_{i}$ may not vary independently but rather relations among them can be derived. For simplicity in the implementation of the developed model, assumptions on the extensibility and curvature field of $\hat{\mathbf{c}}^{i}\left(\zeta_{1}\right)$ are taken such that the overall deformation of a smooth fold becomes a function of the fold angle $\hat{\theta}_{i}$ and the fold width $\hat{w}_{i}^{0}$ (i.e. $\hat{w}_{i}=\hat{w}_{i}\left(\hat{\theta}_{i}, \hat{w}_{i}^{0}\right)$, $\left.\hat{a}_{i}=\hat{a}_{i}\left(\hat{\theta}_{i}, \hat{w}_{i}^{0}\right)\right)$. This process is presented in detail in Ref. [9].

\footnotetext{
${ }^{6}$ A detailed step-by-step proof is provided in Ref. [9].
}

\section{Modeling the Elastic Response of Origami Structures with Smooth Folds}

A plate structural representation is used to model the elastic response of origami structures with smooth folds. The sheet surface $\mathcal{S}_{0}$ corresponds to the mid-surface of the plate. The second-order linearized strain tensor $\varepsilon$ is used as the measure for non-rigid deformation [44]. As previously stated, the fold intersections are not considered herein for the sake of simplification. Thus, the only regions that may undergo non-rigid deformation are the smooth folds.

The tensorial components for each smooth fold are expressed in their associated coordinate system with basis $\left\{\hat{\mathbf{e}}_{1}^{i}, \hat{\mathbf{e}}_{2}^{i}, \hat{\mathbf{e}}_{3}^{i}\right\}$ (defined in Section 3.1). Adopting the Classical Plate Theory [45], the strain is assumed to linearly vary through the plate thickness and the only non-zero components of $\varepsilon$ are additively decomposed as follows:

$$
\left[\begin{array}{l}
\varepsilon_{11} \\
\varepsilon_{22} \\
\varepsilon_{12}
\end{array}\right]=\left[\begin{array}{l}
\varepsilon_{11_{0}} \\
\varepsilon_{22_{0}} \\
\varepsilon_{12_{0}}
\end{array}\right]+z\left[\begin{array}{l}
\kappa_{11} \\
\kappa_{22} \\
\kappa_{12}
\end{array}\right],
$$

where $\varepsilon_{m n_{0}}$ are the in-plane strains at the mid-surface, $\kappa_{m n}$ are the curvatures of the mid-surface, and $z \in$ $\left[-h_{i} / 2, h_{i} / 2\right]$ is the coordinate through the thickness of the plate where $h_{i}$ is the thickness of the $i^{\text {th }}$ smooth fold.

The material comprising the folds is assumed isotropic, homogeneous, and exhibiting linear elastic behavior. Assuming plane stress conditions, the only non-zero components of the second-order Cauchy stress tensor $\boldsymbol{\sigma}$ are obtained through the following relation:

$$
\left[\begin{array}{l}
\sigma_{11} \\
\sigma_{22} \\
\sigma_{12}
\end{array}\right]=\frac{E_{i}}{1-\nu_{i}^{2}}\left[\begin{array}{ccc}
1 & \nu_{i} & 0 \\
\nu_{i} & 1 & 0 \\
0 & 0 & 1-\nu_{i}
\end{array}\right]\left[\begin{array}{l}
\varepsilon_{11} \\
\varepsilon_{22} \\
\varepsilon_{12}
\end{array}\right],
$$

where $E_{i}$ and $\nu_{i}$ are respectively the Young's modulus and the Poisson's ratio of the material comprising the $i^{\text {th }}$ smooth fold.

The total potential energy of the structure, $\Pi$, is given as follows $[44,46,47]$ :

$$
\Pi=U-W_{e},
$$

where $U$ is the elastic strain energy stored in the plate and $W_{e}$ is the external work exerted by body forces and boundary tractions.

For the adopted plane stress assumptions, the elastic strain energy is given as follows:

$$
\begin{aligned}
U & =\int_{\Omega} \frac{1}{2}\left(\sigma_{11} \varepsilon_{11}+\sigma_{22} \varepsilon_{22}+2 \sigma_{12} \varepsilon_{12}\right) \mathrm{d} v, \\
& =\sum_{i=1}^{N_{\mathcal{F}}} \int_{\Omega_{i}} \frac{1}{2}\left(\sigma_{11} \varepsilon_{11}+\sigma_{22} \varepsilon_{22}+2 \sigma_{12} \varepsilon_{12}\right) \mathrm{d} v,
\end{aligned}
$$

where $\Omega$ is the volume of the entire plate domain and $\Omega_{i}$ is the volume of the $i^{\text {th }}$ smooth fold region. 
Inextensional deformation is assumed for the smooth fold regions; therefore, the only contribution to the strain is due to the curvature of $\hat{\mathbf{c}}^{i}\left(\zeta_{1}\right)$, denoted $\kappa\left(\hat{\theta}_{i}\right)$, which in this work is assumed uniform throughout $\hat{\mathbf{c}}^{i}\left(\zeta_{1}\right)$ :

$$
\varepsilon_{22}=z \kappa\left(\hat{\theta}_{i}\right), \quad \varepsilon_{11}=\varepsilon_{12}=0 \quad \forall i=1, \ldots, N_{\mathcal{F}} .
$$

Substituting Eq. (32) into Eq. (29), the following stress components are obtained:

$$
\begin{aligned}
\sigma_{11} & =\frac{\nu_{i} E_{i}}{1-\nu_{i}^{2}} z \kappa\left(\hat{\theta}_{i}\right) \\
\sigma_{22} & =\frac{E_{i}}{1-\nu_{i}^{2}} z \kappa\left(\hat{\theta}_{i}\right) \quad \forall i=1, \ldots, N_{\mathcal{F}} . \\
\sigma_{12} & =0
\end{aligned}
$$

Substituting Eqs. (32) and (33) into Eq. (31), the elastic strain energy of the entire structure is given as follows:

$$
U=\sum_{i=1}^{N_{\mathcal{F}}} \int_{\Omega_{i}} \frac{1}{2} \frac{E_{i}}{1-\nu_{i}^{2}} z^{2} \kappa^{2}\left(\hat{\theta}_{i}\right) \mathrm{d} v
$$

Performing integration over the volume of each smooth fold region to determine $U$, the following is obtained:

$$
\begin{aligned}
U & =\sum_{i=1}^{N_{\mathcal{F}}} \frac{1}{2} \frac{E_{i}\left\|\mathbf{h}^{i}\right\| \hat{w}_{i}^{0}}{1-\nu_{i}^{2}} \int_{-h_{i} / 2}^{h_{i} / 2} z^{2} \kappa^{2}\left(\hat{\theta}_{i}\right) \mathrm{d} z \\
& =\sum_{i=1}^{N_{\mathcal{F}}} \frac{1}{2} \frac{E_{i} I_{i} \hat{w}_{i}^{0}}{1-\nu_{i}^{2}} \kappa^{2}\left(\hat{\theta}_{i}\right),
\end{aligned}
$$

where $I_{i}$ is the area moment of inertia of the $i^{\text {th }}$ smooth fold region:

$$
I_{i}=\frac{1}{12}\left\|\mathbf{h}^{i}\right\| h_{i}^{3}
$$

Only deformation-independent point loads are considered herein. Therefore, the work done by externally applied loads $W_{e}$ is expressed as follows:

$$
W_{e}=\sum_{i=1}^{N_{f}} \mathbf{f}^{i} \cdot \mathbf{u}^{i}(\hat{\boldsymbol{\theta}}),
$$

where $N_{f}$ is the number of applied point loads, $\mathbf{f}^{i} \in \mathbb{R}^{3}$ is the force vector, and $\mathbf{u}^{i}(\hat{\boldsymbol{\theta}}) \in \mathbb{R}^{3}$ is the displacement at the point of application of $\mathbf{f}^{i}$.

By the principle of minimum potential energy, among all possible kinematically admissible displacement fields, the total potential energy $\Pi$ is rendered stationary by the displacement field that allows for the satisfaction of mechanical equilibrium $[44,48]$. In the present model, the displacement field is defined by the fold angles $\hat{\theta}_{i}, i=1, \ldots, N_{\mathcal{F}}$. Therefore, to determine the configuration of an origami structure with smooth folds under applied mechanical loads, the goal is to determine the set of fold angles that minimizes $\Pi$ while satisfying the kinematic constraints stated in Eq. (27):

Find $\hat{\boldsymbol{\theta}}$ that

$$
\begin{array}{ll}
\text { Minimizes } & \Pi(\hat{\boldsymbol{\theta}}) \\
\text { Subject to } & R_{23}^{i}(\hat{\boldsymbol{\theta}})=0, R_{31}^{i}(\hat{\boldsymbol{\theta}})=0, R_{12}^{i}(\hat{\boldsymbol{\theta}})=0, \\
& d_{1}^{i}(\hat{\boldsymbol{\theta}})=0, d_{2}^{i}(\hat{\boldsymbol{\theta}})=0, d_{3}^{i}(\hat{\boldsymbol{\theta}})=0 \\
& \forall i \in\left\{1, \ldots, N_{\mathcal{I}}\right\}
\end{array}
$$

In order to solve the optimization problem of Eq. (38), a penalty method is utilized [49]. In this method, the following auxiliary function $\mathfrak{L}(\hat{\boldsymbol{\theta}})$ is constructed:

$$
\begin{aligned}
\mathfrak{L}(\hat{\boldsymbol{\theta}})= & \Pi(\hat{\boldsymbol{\theta}}) \\
& +\lambda_{R} \sum_{i=1}^{N_{\mathcal{I}}}\left(\left(R_{23}^{i}(\hat{\boldsymbol{\theta}})\right)^{2}+\left(R_{31}^{i}(\hat{\boldsymbol{\theta}})\right)^{2}+\left(R_{12}^{i}(\hat{\boldsymbol{\theta}})\right)^{2}\right) \\
& +\lambda_{d} \sum_{i=1}^{N_{\mathcal{I}}}\left(\left(d_{1}^{i}(\hat{\boldsymbol{\theta}})\right)^{2}+\left(d_{2}^{i}(\hat{\boldsymbol{\theta}})\right)^{2}+\left(d_{3}^{i}(\hat{\boldsymbol{\theta}})\right)^{2}\right),
\end{aligned}
$$

where $\lambda_{R} \in \mathbb{R}_{>0}$ and $\lambda_{d} \in \mathbb{R}_{>0}$ are the penalty scalars. The configuration that minimizes $\Pi$ while satisfying the kinematic constraints meets the following optimality conditions:

$$
\frac{\partial \mathfrak{L}}{\partial \hat{\theta}_{i}}=0 \quad \forall i \in\left\{1, \ldots, N_{\mathcal{F}}\right\} .
$$

\section{Numerical Implementation}

The mechanical loading applied to the origami structures with smooth folds is evolved in various loading increments. For each load increment, the goal is to find the configuration that satisfies the optimality conditions of Eq. (40).

The residual vector $\mathfrak{R} \in \mathbb{R}^{N_{\mathcal{F}}}$ with components $\mathfrak{R}_{i}$ is first defined:

$$
\mathfrak{R}_{i}(\hat{\boldsymbol{\theta}}):=\frac{\partial \mathfrak{L}(\hat{\boldsymbol{\theta}})}{\partial \hat{\theta}_{i}} .
$$

The present implementation iteratively solves for increments in fold angles $\hat{\boldsymbol{\theta}}$ that minimize the components of the vector $\mathfrak{R}(\hat{\boldsymbol{\theta}})$, which must be zero (or sufficiently close to zero by introducing a numerical tolerance) for a configuration to minimize $\Pi(\hat{\boldsymbol{\theta}})$ subject to the kinematic constraints of Eq. (27) (see Eq. (40)). Following the Newton's method, the first-order expansion of $\mathfrak{R}$ is used to find the increment in $\hat{\boldsymbol{\theta}}$ required to minimize $\mathfrak{R}$ :

$$
\mathfrak{R}(\hat{\boldsymbol{\theta}}+\Delta \hat{\boldsymbol{\theta}})=\mathfrak{R}(\hat{\boldsymbol{\theta}})+\frac{\partial \boldsymbol{R}(\hat{\boldsymbol{\theta}})}{\partial \hat{\boldsymbol{\theta}}} \Delta \hat{\boldsymbol{\theta}}+\ldots=\mathbf{0}_{N_{\mathcal{F}}}
$$

Therefore, the following correction increment $\Delta \hat{\boldsymbol{\theta}}$ is obtained from the first-order expansion: 


$$
\Delta \hat{\boldsymbol{\theta}}=-\left(\frac{\partial \mathfrak{R}(\hat{\boldsymbol{\theta}})}{\partial \hat{\boldsymbol{\theta}}}\right)^{-1} \mathfrak{R}(\hat{\boldsymbol{\theta}}) .
$$

At the $l^{\text {th }}$ loading increment, the fold angles are corrected iteratively using the following correction increment:

$$
{ }_{l}^{k} \Delta \hat{\boldsymbol{\theta}}=-\left(\frac{\partial \mathfrak{R}\left({ }_{l}^{k} \hat{\boldsymbol{\theta}}\right)}{\partial \hat{\boldsymbol{\theta}}}\right)^{-1} \mathfrak{R}\left({ }_{l}^{k} \hat{\boldsymbol{\theta}}\right),
$$

where the subscripts in the previous equation refer to the load increment number and the superscripts to the fold angle correction iteration number. After ${ }_{l}^{k} \Delta \hat{\boldsymbol{\theta}}$ is obtained using Eq. (44), the fold angles are updated as follows:

$$
{ }_{l}^{k+1} \hat{\boldsymbol{\theta}}={ }_{l}^{k} \hat{\boldsymbol{\theta}}+{ }_{l}^{k} \Delta \hat{\boldsymbol{\theta}} .
$$

The fold angle correction process of Eqs. and (45) is repeated for each loading increment until $\left\|\boldsymbol{R}\left({ }_{l}^{k+1} \hat{\boldsymbol{\theta}}\right)\right\| / N_{\mathcal{F}}<$ tol1 or $\left\|{ }_{l}^{k} \Delta \hat{\boldsymbol{\theta}}\right\| / N_{\mathcal{F}}<$ tol2, where tol1 and tol2 are numerical tolerances. Table 1 summarizes the numerical procedure followed to simulate origami structures with smooth folds subject to mechanical loading.

Table 1: Numerical procedure followed to simulate origami structures with smooth folds at the $l^{\text {th }}$ load increment.

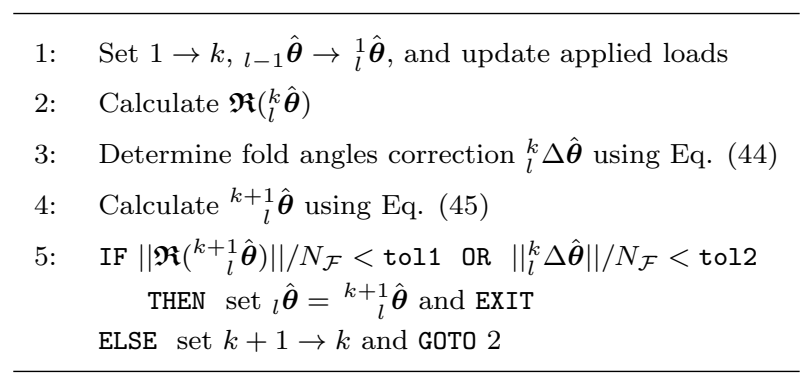

\section{Finite Element Analysis}

Finite element analysis (FEA) is used for numerical validation of the model for origami with smooth elastic folds. In this work, FEA is performed using Abaqus finite element software. Static analysis steps considering large rotations are used. Abaqus S4 elements (4-node doubly curved general-purpose shells, finite membrane strains) are used to discretize the sheet domain.

\section{Experimental Analysis}

In addition to numerical validation, experimental validation of the proposed model for origami with smooth elastic folds is also considered. The experimental testing is performed using 3D-printed samples fabricated using the desktop 3D printer LulzBot ${ }^{\circledR}$ TAZ 5. Pictures of the
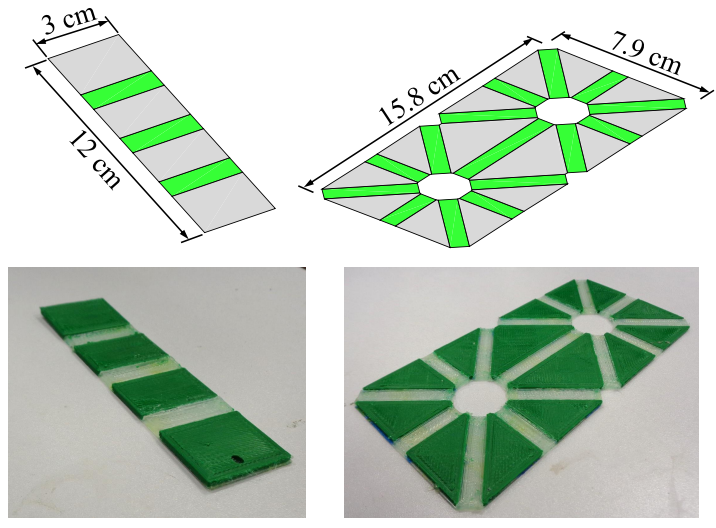

Figure 12: Samples used for experimental validation and their corresponding source models and dimensions. Left: Sheet with no fold intersections. Right: Sheet with two fold intersections.

considered 3D-printed samples and their associated source model and dimensions are shown in Fig. 12.

It is required for experimental validation to fabricate sheets with compliant regions corresponding to the smooth folds and stiff regions corresponding to the rigid faces. In view of this, the smooth folds regions are printed from NinjaFlex ${ }^{\circledR}$ elastomeric material and the face regions are printed from ABS plastic. The material parameters for these two materials are provided in Table 2. The Young's modulus of ABS plastic $\left(E_{A P}\right)$ is two orders of magnitude higher than the Young's modulus of NinjaFlex ${ }^{\circledR}\left(E_{N F}\right)$. Both values were obtained from the literature $[50,51]$. The Poisson's ratio of the two materials $\left(\nu_{A P}\right.$ and $\left.\nu_{N F}\right)$ and their mass density $\left(\rho_{A P}\right.$ and $\left.\rho_{N F}\right)$ are also required for analysis and provided in Table 2 . The out-of-plane thickness of the smooth fold regions in the fabricated samples is approximately $1.8 \mathrm{~mm}$ while that of the face regions is approximately $2.0 \mathrm{~mm}$.

Table 2: Material parameters used for the modeling of origami sheets with elastic smooth folds [50, 51].

\begin{tabular}{llll}
\hline Parameter & Value & Parameter & Value \\
\hline$E_{A P}$ & $2.41 \mathrm{GPa}$ & $E_{N F}$ & $15.2 \mathrm{MPa}$ \\
$\nu_{A P}$ & 0.35 & $\nu_{N F}$ & 0.45 \\
$\rho_{A P}$ & $1040 \mathrm{~kg} / \mathrm{m}^{3}$ & $\rho_{N F}$ & $1200 \mathrm{~kg} / \mathrm{m}^{3}$ \\
\hline
\end{tabular}

The utilized experimental setup is shown in Fig. 13. One face region of the samples is fixed by clamping it. Holes are perforated at selected faces of the samples in the locations where loads are applied. Then, weights are connected to the samples using strings knotted through the holes in order to represent point loads. The out-of-plane positions of the points of interest under various loading conditions are measured using a vertically aligned ruler. The smallest length unit measured by the ruler is $1 \mathrm{~mm}$ and thus there is an instrumental uncertainty in the measured positions 
of $\pm 0.5 \mathrm{~mm}$. As will be shown in the subsequent section, the measured displacements of the points of interest are in the order of centimeters and therefore the instrumental uncertainty did not play a significant role in the reported experimental results.

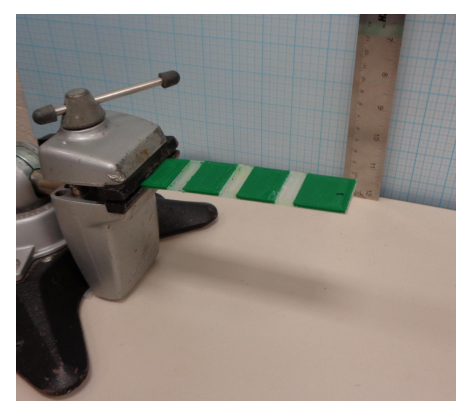

Figure 13: Experimental setup for the mechanical testing of origami sheets.

\section{Results}

\subsection{Experimental Validation}

The considered boundary value problems for numerical and experimental validation are shown in Fig. 14. Mechanical loads include applied point loads and the weight of the sheets. The gravity vector is given as $\mathbf{g}=[0,0,-g]^{\mathrm{T}}$ (with $g=9.81 \mathrm{~m} / \mathrm{s}^{2}$ ). The body force associated with the weight of each region is modeled as a point load applied at its corresponding center of mass and with a force vector of $\mathbf{g}$ multiplied by the total mass of the region. The blue dot in the schematics shown in Fig. 14 indicate the points where the out-of-plane displacement is measured.
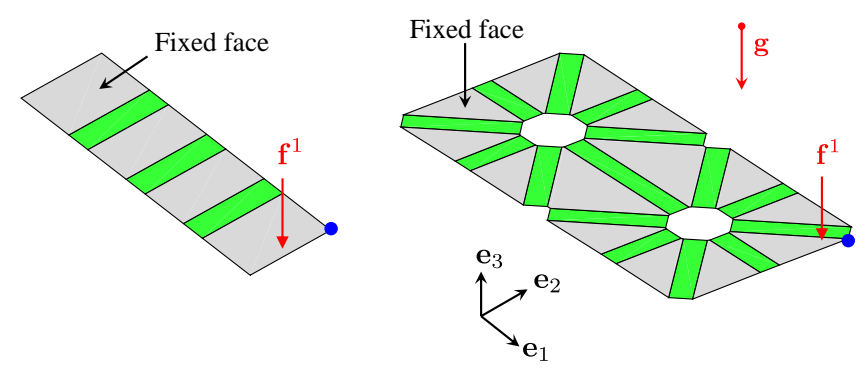

Figure 14: Boundary value problems considered where $\mathbf{f}_{1}=[0,0, f]^{\mathrm{T}}$. The blue dot indicates the point where the out-of-plane displacement is measured.

Figure 15 shows the out-of-plane displacement at the point of interest vs. applied load $f$ (see Fig. 14) for the two samples. It is found experimentally that gravity has a negligible effect on the deformation of the sheet with no fold intersections and it is neglected in the simulations using FEA and the model for origami with smooth folds. Figure 15(a) shows that there is an excellent agreement among the results of the experimental, FEA, and the present model analyses for the sheet without fold intersections. The configurations of this sample under the same loading conditions as obtained using the various analyses are shown in Fig. 16.
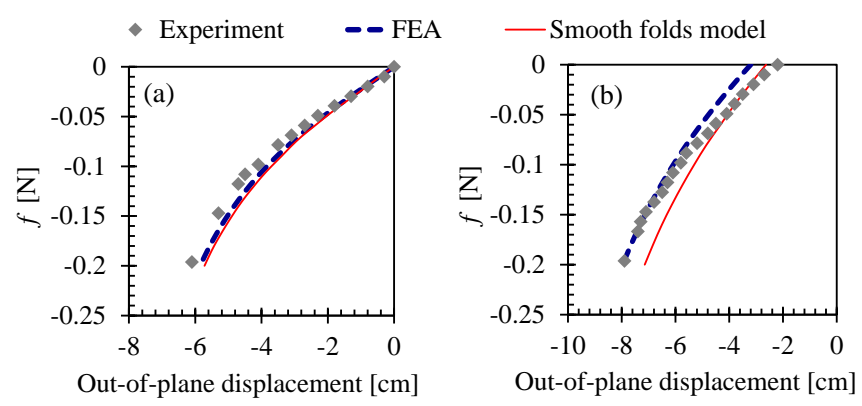

Figure 15: Load vs. out-of-plane displacement for (a) Sheet with no fold intersections and (b) Sheet with two fold intersections. (a)

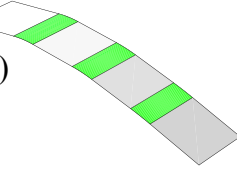

(b)

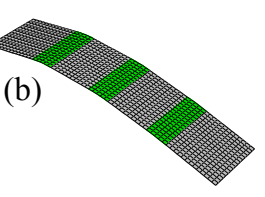

(c)

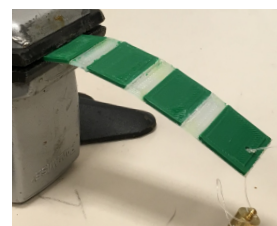

Figure 16: Configurations of a sheet without fold intersections under an applied point load of approximately $0.1 \mathrm{~N}$ : (a) Origami with smooth folds model, (b) FEA, and (c) Experimental.

Slight discrepancies among the results of the FEA, experimental, and the present model analyses for the sheet with two fold intersections are observed in Fig. 15(b). Deformed configurations of this sample as obtained from the different analyses under the same loading conditions are shown in Fig. 17. It is expected that the results from FEA match the experimental results slightly better than the present model because in FEA the deformation of the folds is not limited to pure bending, but the folds can also stretch, twist, etc. This is in contrast to the pure bending/constant curvature deformation at the folds regions assumed for simplicity in the model for origami with smooth folds. Other causes of the observed discrepancies include the possible difference between the assumed material parameters from the literature and the actual material parameters of the sheets. Also, it was observed that the interfaces between the fold and face regions in the experimental samples were slightly damaged at certain locations. This could make the response of the experimental samples more compliant than the response predicted by the computational analyses which assume perfect bonding between the various sheet regions.

\subsection{Case Studies}

The purpose of this section is to study the performance of the proposed model in terms of comparison of results 
(a)

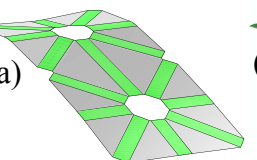

(b)
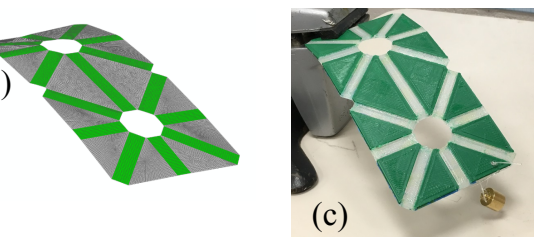

Figure 17: Sheet with two fold intersections under gravity load and an applied point load of approximately $0.1 \mathrm{~N}$ : (a) Origami with smooth folds model, (b) FEA, and (c) Experimental.

with higher-fidelity FEA simulations and computational efficiency. Unless otherwise noted, the material parameters of NinjaFlex ${ }^{\circledR}$ and ABS plastic provided in Tab. 2 are respectively assumed for the smooth fold regions and the face regions.

The various cases shown in Figs. 18-21 consider different fold widths, material parameters, mechanical loading, and fold pattern geometry. There is an excellent agreement in the deformation predicted using FEA and the model for origami with smooth folds for all the cases. This further shows the capability of the proposed model to capture realistic structural behavior of origami structures having elastic smooth folds.

The computational efficiency of the proposed model for origami with smooth folds as compared to FEA is also explored. It is noted that all simulations are performed in the same computer and that the model for origami with smooth folds is implemented in Matlab. Table 3 shows the wall-clock time in seconds required for each case study shown in Figs. 18-21. The wall-clock time required for analysis using the proposed model is significantly lower than that required using FEA for all cases (by more than $30 \%$ except for case 4). This shows that the proposed model provides accurate results as compared to higherfidelity FEA while being more computationally efficient.

Table 3: Wall-clock time in seconds required for each case study.

\begin{tabular}{llll}
\hline Case study & $\begin{array}{l}\text { Smooth folds } \\
\text { model }\end{array}$ & FEA & Difference (\%) \\
\hline Case 1 (Fig. 18(b)) & 426 & 944 & 54.9 \\
Case 2 (Fig. 18(c)) & 395 & 601 & 34.3 \\
Case 3 (Fig. 19) & 401 & 655 & 38.8 \\
Case 4 (Fig. 20) & 448 & 544 & 17.6 \\
Case 5 (Fig. 21) & 449 & 886 & 49.3 \\
\hline
\end{tabular}

\section{Summary and Conclusions}

A novel model for the structural response of origami structures with smooth folds was presented in this paper. The model is divided into kinematic equations and structural analysis equations. The key equations from kinematics are parameterization of the smooth folds (Eq. (1)) which define these regions as ruled surfaces of prismatic

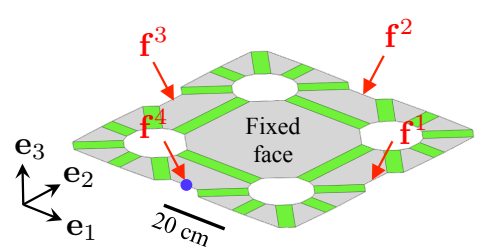

(a)

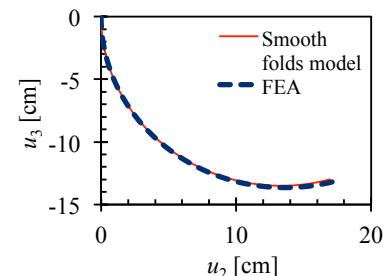

(b)

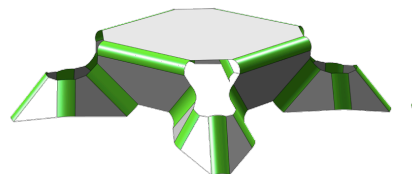

(d)

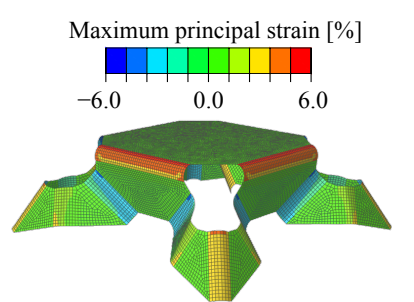

(f)

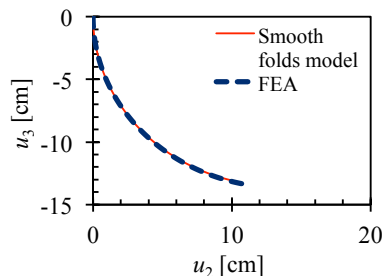

(c)

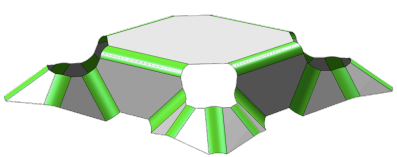

(e)

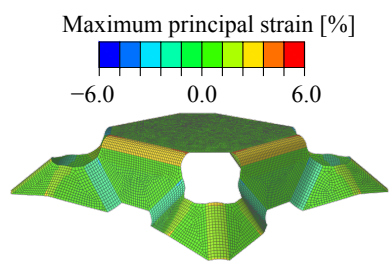

(g)
Figure 18: Case 1: (a) Boundary value problem. The components of the force vectors are proportionally increased from $\mathbf{0}_{3}$ to the following values: $\mathbf{f}^{1}=[-2,0,-1.2]^{\mathrm{T}} \mathrm{N}, \mathbf{f}^{2}=[0,-2,-1.2]^{\mathrm{T}} \mathrm{N}$, $\mathbf{f}^{3}=[2,0,-1.2]^{\mathrm{T}} \mathrm{N}$, and $\mathbf{f}^{4}=[0,2,-1.2]^{\mathrm{T}} \mathrm{N}$. The blue dot indicates the point for which displacements are compared. (b) Comparison of displacements of a particular point determined using the model for origami with smooth folds and FEA. (d) Current configuration at maximum loading for the model of origami with smooth folds. (f) Current configuration at maximum loading for FEA showing maximum principal strain. Case 2: Results of the boundary value problem shown in (a) with folds having a Young's modulus value of $2 E_{N F}$ are presented in (c), (e), and (g).

cross-section, and the constraints on the fold shape variables that allow for valid configurations (Eqs. (24) and $(25))$.

The elastic response is modeled by first assuming a strain field at the smooth folds that is a function of the stretching and curvature of the parametric curve defining the fold cross-section. By applying the principle of minimum potential energy, kinematically admissible displacement fields that satisfy mechanical equilibrium under applied loads are determined. A numerical implementation of this model is described and results for various boundary value problems are compared with higher-fidelity FEA and experimental data. Good agreement is observed among 


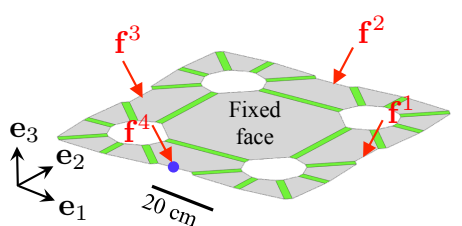

(a)

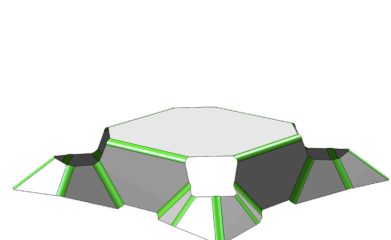

(c)

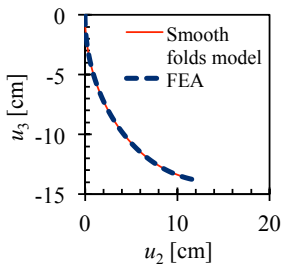

(b)

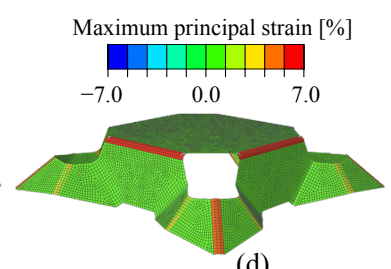

(d)
Figure 19: Case 3: (a) Boundary value problem. All the specifications of the boundary value problem of Case 1 are applicable except that the width of the folds is reduced by one-half for this case. (b) Comparison of displacements of a particular point determined using the model for origami with smooth folds and FEA. (c) Current configuration at maximum loading for the model of origami with smooth folds. (d) Current configuration at maximum loading for FEA showing maximum principal strain.

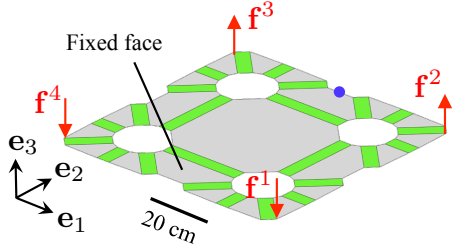

(a)

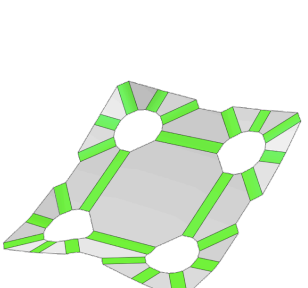

(c)

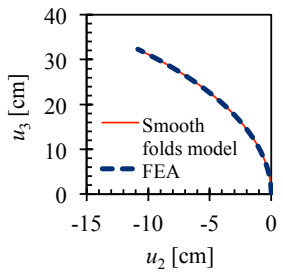

(b)

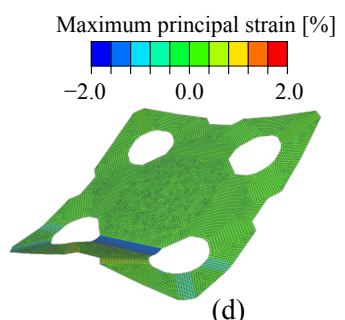

Figure 20: Case 4: (a) Boundary value problem. All the specifications of the boundary value problem of Case 1 are applicable except for the applied boundary conditions. The components of the force vectors are proportionally increased from $\mathbf{0}_{3}$ to the following values: $\mathbf{f}^{1}=[0,0,-0.11]^{\mathrm{T}} \mathrm{N}, \mathbf{f}^{2}=[0,0,0.05]^{\mathrm{T}} \mathrm{N}, \mathbf{f}^{3}=[0,0,0.05]^{\mathrm{T}} \mathrm{N}$, and $\mathbf{f}^{4}=[0,0,-0.11]^{\mathrm{T}} \mathrm{N}$. The blue dot indicates the point for which displacements are compared. (b) Comparison of displacements of a particular point determined using the model for origami with smooth folds and FEA. (c) Current configuration at maximum loading for the model of origami with smooth folds. (d) Current configuration at maximum loading for FEA showing maximum principal strain.

the various analyses. The present model allows for the simulation of origami structures having arbitrary patterns of elastic smooth folds. As such, it can be utilized for the

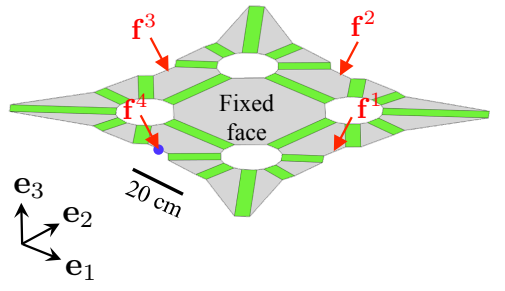

(a)

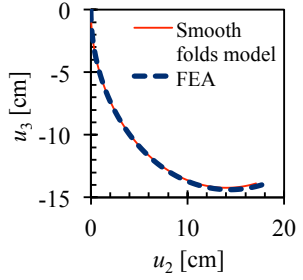

(b)

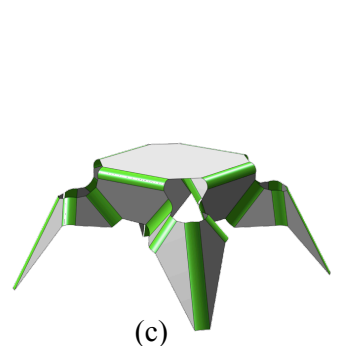

(c)

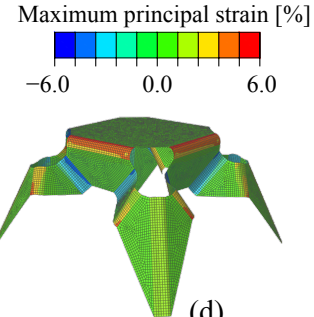

(d)
Figure 21: Case 5: (a) Boundary value problem. The fold pattern is obtained by altering the position of the corner vertices in the fold pattern of Case 1. The boundary conditions, fold widths, and material parameters are those of Case 1. (b) Comparison of displacements of a particular point determined using the model for origami with smooth folds and FEA. (c) Current configuration at maximum loading for the model of origami with smooth folds. (d) Current configuration at maximum loading for FEA showing maximum principal strain.

design of origami-based mechanisms and structures.

\section{Acknowledgments}

This work is supported by the National Science Foundation and the Air Force Office of Scientific Research under grant EFRI-1240483. Any opinions, findings, conclusions or recommendations are those of the authors and do not necessarily reflect the views of the U.S. Air Force. The authors would like to acknowledge Mr. Doe Young Hur for fabricating the experimental samples and Mr. Cullen Nauck for performing the experiments. Finite element analysis was performed using a research license granted by Simulia.

\section{References}

[1] E. D. Demaine, Folding and unfolding linkages, paper, and polyhedra, in: Discrete and Computational Geometry, Springer, 2001, pp. 113-124.

[2] B. A. Cipra, In the fold: Origami meets mathematics, SIAM News 34 (8) (2001) 1-4.

[3] T. Tarnai, Origami in structural engineering, in: IASS Symposium 2001: International Symposium on Theory, Design and Realization of Shell and Spatial Structures, Nagoya, Japan, 9-13 Oct. 2001, 2001, pp. 298-299.

[4] C. Cromvik, K. Eriksson, Airbag folding based on origami mathematics, in: Origami 4, 4th International Meeting of Origami Science, Mathematics, and Education, 2006, pp. 129-139.

[5] E. Hawkes, B. An, N. M. Benbernou, H. Tanaka, S. Kim, E. D. Demaine, D. Rus, R. J. Wood, Programmable matter by folding, Proceedings of the National Academy of Sciences 107 (28) (2010) 12441-12445. 
[6] S. Pandey, E. Gultepe, D. H. Gracias, Origami inspired selfassembly of patterned and reconfigurable particles, Journal of Visualized Experiments: JoVE (72).

[7] L. J. Fei, D. Sujan, Origami theory and its applications: A literature review, International Journal of Social, Behavioral, Educational, Economic and Management Engineering 7 (1) (2013) $113-117$.

[8] E. A. Peraza-Hernandez, D. J. Hartl, R. J. Malak Jr., D. C. Lagoudas, Origami-inspired active structures: a synthesis and review, Smart Materials and Structures 23 (9) (2014) 094001.

[9] E. A. Peraza Hernandez, D. J. Hartl, D. C. Lagoudas, Kinematics of origami structures with smooth folds, To be submitted.

[10] T. Tachi, Simulation of rigid origami, Origami 4, 4th International Meeting of Origami Science, Mathematics, and Education (2009) 175-187.

[11] Z. Abel, J. Cantarella, E. D. Demaine, D. Eppstein, T. C. Hull, J. S. Ku, R. J. Lang, T. Tachi, Rigid origami vertices: conditions and forcing sets, Journal of Computational Geometry 7 (1) (2016) 171-184.

[12] X.-Y. Li, T. Ju, Y. Gu, S.-M. Hu, A geometric study of V-style pop-ups: Theories and algorithms, in: ACM SIGGRAPH 2011 Papers, SIGGRAPH '11, ACM, New York, NY, USA, 2011, pp. 98:1-98:10.

[13] S. N. Le, S.-J. Leow, T.-V. Le-Nguyen, C. Ruiz, K.-L. Low, Surface and contour-preserving origamic architecture paper popups, IEEE Transactions on Visualization and Computer Graphics 20 (2) (2014) 276-288.

[14] T. A. Evans, R. J. Lang, S. P. Magleby, L. L. Howell, Rigidly foldable origami gadgets and tessellations, Royal Society Open Science $2(9)$.

[15] T. Tachi, Geometric considerations for the design of rigid origami structures, in: Proceedings of the International Association for Shell and Spatial Structures (IASS) Symposium, Vol. 12, 2010, pp. 458-460.

[16] M. Schenk, S. D. Guest, Origami folding: A structural engineering approach, Origami 5: Fifth International Meeting of Origami Science, Mathematics, and Education (2011) 291-304.

[17] S. Pellegrino, C. Calladine, Matrix analysis of statically and kinematically indeterminate frameworks, International Journal of Solids and Structures 22 (4) (1986) $409-428$.

[18] S.-M. Belcastro, T. C. Hull, Modelling the folding of paper into three dimensions using affine transformations, Linear Algebra and its Applications 348 (13) (2002) $273-282$.

19] S.-M. Belcastro, T. C. Hull, A mathematical model for non-flat origami, in: Origami3: Proc. the 3rd International Meeting of Origami Mathematics, Science, and Education, 2002, pp. 39-51.

[20] T. Tachi, Rigid origami simulator, http://www.tsg.ne.jp/TT/software/.

[21] T. Tachi, Freeform origami, http://www.tsg.ne.jp/TT/software/.

[22] T. Tachi, Freeform variations of origami, J. Geom. Graph 14 (2) (2010) 203-215.

[23] E. Demaine, M. Demaine, D. Koschitz, T. Tachi, Curved crease folding: a review on art, design and mathematics, in: Proceedings of the IABSE-IASS Symposium: Taller, Longer, Lighter (IABSE-IASS2011), London, England, Sept, Citeseer, 2011, pp. 20-23.

[24] E. D. Demaine, M. L. Demaine, D. A. Huffman, D. Koschitz, T. Tachi, Characterization of curved creases and rulings: Design and analysis of lens tessellations, Origami6: I. Mathematics (2015) 209.

[25] M. A. Dias, L. H. Dudte, L. Mahadevan, C. D. Santangelo, Geometric mechanics of curved crease origami, Physical Review Letters 109 (11) (2012) 114301

[26] J. L. Silverberg, A. A. Evans, L. McLeod, R. C. Hayward, T. Hull, C. D. Santangelo, I. Cohen, Using origami design principles to fold reprogrammable mechanical metamaterials, Science 345 (6197) (2014) 647-650.

[27] K. C. Francis, L. T. Rupert, R. J. Lang, D. C. Morgan, S. P. Magleby, L. L. Howell, From crease pattern to product: Considerations to engineering origami-adapted designs, in: ASME 2014 IDETC-CIE, American Society of Mechanical Engineers,
2014, pp. Paper No. DETC2014-34031, pp. V05BT08A030.

[28] E. A. Peraza Hernandez, S. Hu, H. W. Kung, D. Hartl, E. Akleman, Towards building smart self-folding structures, Computers \& Graphics 37 (6) (2013) 730-742.

[29] E. A. Peraza-Hernandez, D. J. Hartl, R. J. M. Jr, Design and numerical analysis of an SMA mesh-based self-folding sheet, Smart Materials and Structures 22 (9) (2013) 094008.

[30] E. A. Peraza Hernandez, D. J. Hartl, R. J. Malak, E. Akleman, O. Gonen, H.-W. Kung, Design tools for patterned self-folding reconfigurable structures based on programmable active laminates, Journal of Mechanisms and Robotics 8 (3) (2016) 031015.

[31] J. Guan, H. He, D. J. Hansford, L. J. Lee, Self-folding of threedimensional hydrogel microstructures, The Journal of Physical Chemistry B 109 (49) (2005) 23134-23137.

[32] Y. L. Kergosien, H. Gotoda, T. L. Kunii, Bending and creasing virtual paper, Computer Graphics and Applications, IEEE 14 (1) (1994) 40-48.

[33] H.-D. Hwang, S.-H. Yoon, Constructing developable surfaces by wrapping cones and cylinders, Computer-Aided Design 58 (2015) $230-235$.

[34] L. Zhu, T. Igarashi, J. Mitani, Soft folding, Computer Graphics Forum 32 (7) (2013) 167-176.

[35] T. Tachi, Interactive form-finding of elastic origami, in: Proceedings of the International Association for Shell and Spatial Structures (IASS) Symposium, 2013.

[36] T. Lozano-Perez, Spatial planning: A configuration space approach, Computers, IEEE Transactions on 100 (2) (1983) 108120.

[37] L. E. Kavraki, P. Švestka, J.-C. Latombe, M. H. Overmars, Probabilistic roadmaps for path planning in high-dimensional configuration spaces, Robotics and Automation, IEEE Transactions on 12 (4) (1996) 566-580

[38] J. L. Gross, T. W. Tucker, Topological graph theory, Courier Corporation, 1987.

39] E. Akleman, J. Chen, J. L. Gross, Block meshes: Topologically robust shape modeling with graphs embedded on 3-manifolds, Computers \& Graphics 46 (2015) 306-326.

[40] T. Tachi, Rigid folding of periodic origami tessellations, Origami 6: I. Mathematics (2015) 97-108.

[41] B. A. Barsky, T. D. DeRose, Geometric continuity of parametric curves, Computer Science Division, University of California, 1984.

[42] B. A. Barsky, R. H. Bartels, J. C. Beatty, An Introduction to Splines for Use in Computer Graphics and Geometric Modeling, Los Altos, Calif.: M. Kaufmann Publishers, 1987.

[43] W. Cheney, D. Kincaid, Numerical Analysis. Mathematics of Scientific Computing, Brooks \& Cole Publishing Company, 1996

[44] W. S. Slaughter, The Linearized Theory of Elasticity, Birkhäuser, Boston, 2002.

[45] J. N. Reddy, Mechanics of Laminated Composite Plates: Theory and Analysis, CRC press, 1997.

[46] J. N. Reddy, Energy Principles and Variational Methods in Applied Mechanics, John Wiley \& Sons, Hoboken NJ, 2002.

[47] E. A. Peraza Hernandez, B. Kiefer, D. J. Hartl, A. Menzel, D. C. Lagoudas, Analytical investigation of structurally stable configurations in shape memory alloy-actuated plates, International Journal of Solids and Structures 69 (2015) 442-458.

[48] O. A. Bauchau, J. I. Craig, Structural Analysis - With Applications to Aerospace Structures, Springer, 2009.

[49] J. N. Reddy, An Introduction to Nonlinear Finite Element Analysis: with applications to heat transfer, fluid mechanics, and solid mechanics, OUP Oxford, 2014

[50] E. A. Peraza Hernandez, D. J. Hartl, A. Kotz, R. J. Malak, Design and optimization of an SMA-based self-folding structural sheet with sparse insulating layers, in: ASME SMASIS 2014, American Society of Mechanical Engineers, 2014, p. V001T01A015.

[51] P. Kulkarni, Centrifugal forming and mechanical properties of silicone-based elastomers for soft robotic actuators, Ph.D. thesis, Rutgers University-Graduate School-New Brunswick (2015). 


\section{Appendix A. Nomenclature}

Table A.4: List of main symbols related to the kinematics. Unless otherwise noted, the physical tensorial components are expressed in the fixed global coordinate system with basis $\left\{\mathbf{e}_{1}, \mathbf{e}_{2}, \mathbf{e}_{3}\right\}$.

\begin{tabular}{|c|c|}
\hline $\mathcal{S}_{0}, \mathcal{S}_{t}$ & Sheet reference/current configuration \\
\hline $\mathcal{P}_{0}^{i}, \mathcal{P}_{t}^{i}$ & Reference/current configuration of the $i^{\text {th }}$ face \\
\hline $\mathcal{F}_{0}^{i}, \mathcal{F}_{t}^{i}$ & $\begin{array}{l}\text { Reference/current configuration of the } i^{\text {th }} \text { smooth } \\
\text { fold }\end{array}$ \\
\hline $\mathcal{I}_{0}^{i}, \mathcal{I}_{t}^{i}$ & $\begin{array}{l}\text { Reference/current configuration of the } i^{\text {th }} \text { fold in- } \\
\text { tersection }\end{array}$ \\
\hline$N_{\mathcal{P}}, N_{\mathcal{F}}, N_{\mathcal{I}}$ & $\begin{array}{l}\text { Number of faces/smooth folds/fold intersections } \\
\text { in the sheet }\end{array}$ \\
\hline $\mathcal{F}^{i}\left(\zeta_{1}, \zeta_{2}\right)$ & Surface parameterization of $\mathcal{F}_{t}^{i}$ \\
\hline $\mathbf{c}^{i}\left(\zeta_{1}\right)$ & Fold cross-section parametric curve \\
\hline $\mathbf{h}^{i}$ & Smooth fold director vector \\
\hline$\hat{\theta}_{i}$ & Fold angle of the $i^{\text {th }}$ smooth fold \\
\hline$\hat{w}_{i}$ & $\begin{array}{l}\text { Distance between the two end-points of the cross- } \\
\text { section curve of the } i^{\text {th }} \text { fold }\end{array}$ \\
\hline$\hat{a}_{i}$ & Asymmetry variable of the $i^{\text {th }}$ smooth fold \\
\hline$\hat{\mathbf{e}}_{1}^{i}, \hat{\mathbf{e}}_{2}^{i}, \hat{\mathbf{e}}_{3}^{i}$ & Fold-attached orthonormal vectors \\
\hline$\hat{\mathbf{c}}^{i}\left(\zeta_{1}\right)$ & $\begin{array}{l}\text { Fold cross-section parametric curve expressed in } \\
\text { the basis }\left\{\hat{\mathbf{e}}_{1}^{i}, \hat{\mathbf{e}}_{2}^{i}, \hat{\mathbf{e}}_{3}^{i}\right\}\end{array}$ \\
\hline $\mathbf{v}^{j}$ & Position vector of the $j^{\text {th }}$ vertex \\
\hline$\hat{\mathbf{v}}^{i 1}, \hat{\mathbf{v}}^{i 2}$ & $\begin{array}{l}\text { Position vectors of the vertices from the which the } \\
i^{\text {th }} \text { fold centerline emanates and ends }\end{array}$ \\
\hline$\hat{\mathbf{p}}_{j}^{i}$ & $\begin{array}{l}j=1,2,3,4 \text {, corner points position vectors of the } \\
i^{\text {th }} \text { smooth fold in the reference configuration }\end{array}$ \\
\hline$\hat{r}_{1}^{i}, \hat{r}_{2}^{i}$ & Length parameters of the $i^{\text {th }}$ smooth fold \\
\hline $\mathbf{m}^{j k}$ & $\begin{array}{l}\text { Vector along the length of the } k^{\text {th }} \text { fold centerline } \\
\text { incident to the } j^{\text {th }} \text { interior vertex that emanates } \\
\text { from such a vertex }\end{array}$ \\
\hline$\varphi(\mathbf{y})$ & 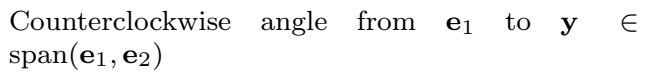 \\
\hline$\theta_{j k}$ & $\begin{array}{l}\text { Fold angle of the } k^{\text {th }} \text { fold adjacent to the } j^{\text {th }} \text { in- } \\
\text { terior fold intersection }\end{array}$ \\
\hline$w_{j k}$ & $\begin{array}{l}\text { Distance between the two end-points of the cross- } \\
\text { section curve of the } k^{\text {th }} \text { fold adjacent to the } j^{\text {th }} \\
\text { interior fold intersection }\end{array}$ \\
\hline$a_{j k}$ & $\begin{array}{l}\text { Asymmetry variable of the } k^{\text {th }} \text { fold adjacent to } \\
\text { the } j^{\text {th }} \text { interior fold intersection }\end{array}$ \\
\hline$\gamma_{j}(\eta)$ & Closed path in the $j^{\text {th }}$ vertex module enclosing $\mathcal{I}_{0}^{j}$ \\
\hline $\mathbf{b}_{L}^{j k}, \mathbf{b}_{R}^{j k}$ & $\begin{array}{l}\text { Position vectors of the points where } \gamma_{j}(\eta) \text { en- } \\
\text { ters/exits the } k^{\text {th }} \text { fold adjacent to the } j^{\text {th }} \text { interior } \\
\text { fold intersection }\end{array}$ \\
\hline $\mathbf{Q}_{i}(\phi)$ & $\begin{array}{l}\text { Transformation matrix in homogeneous coordi- } \\
\text { nates associated with a rotation by } \phi \text { about an } \\
\text { axis aligned to } \mathbf{e}_{i}\end{array}$ \\
\hline $\mathbf{T}(\mathbf{b})$ & $\begin{array}{l}\text { Transformation matrix in homogeneous coordi- } \\
\text { nates associated with a translation by vector } \mathbf{b}\end{array}$ \\
\hline $\mathbf{R}_{i}(\phi)$ & $\begin{array}{l}\text { Transformation matrix associated with a rotation } \\
\text { by } \phi \text { about an axis aligned to } \mathbf{e}_{i}\end{array}$ \\
\hline $\mathbf{L}^{j k}$ & $\begin{array}{l}\text { Transformation matrix associated with the } k^{\text {th }} \\
\text { fold adjacent to the } j^{\text {th }} \text { interior fold intersection }\end{array}$ \\
\hline $\mathbf{g}^{j k}$ & $\begin{array}{l}\text { Recursively determined vectors accounting for the } \\
\text { change in the distance between the smooth folds } \\
\text { boundary rulings in a current configuration }\end{array}$ \\
\hline $\mathbf{w}^{j k}, \mathbf{l}^{j k}$ & $\begin{array}{l}\text { Vectors with start-points and end-points corre- } \\
\text { sponding to the points where the path } \gamma_{j}(\eta) \\
\text { crosses the boundary rulings of the smooth folds } \\
\text { (see Eqs. (19) and }(20) \text { ) }\end{array}$ \\
\hline$\tilde{\mathbf{w}}^{j k}, \tilde{\mathbf{l}}^{j k}$ & Vectors $\mathbf{w}^{j k}, \mathbf{l}^{j k}$ rotated by $-\varphi\left(\mathbf{m}^{j k}\right)$ about $\mathbf{e}_{3}$ \\
\hline $\mathbf{X}, \mathbf{x}$ & $\begin{array}{l}\text { Position vectors of a point in a face at the refer- } \\
\text { ence/current configuration }\end{array}$ \\
\hline $\mathbf{R}^{j}$ & Rotation constraint matrix \\
\hline $\mathbf{d}^{j}$ & Translation constraint vector \\
\hline
\end{tabular}

Table A.5: List of main symbols related to the structural analysis. Unless otherwise noted, the physical tensorial components are expressed in the fixed global coordinate system with basis $\left\{\mathbf{e}_{1}, \mathbf{e}_{2}, \mathbf{e}_{3}\right\}$.

\begin{tabular}{|c|c|}
\hline$\sigma_{m n}$ & $\begin{array}{l}\text { Components of the Cauchy stress tensor in the basis } \\
\left\{\hat{\mathbf{e}}_{1}^{i}, \hat{\mathbf{e}}_{2}^{i}, \hat{\mathbf{e}}_{3}^{i}\right\}\end{array}$ \\
\hline$\varepsilon_{m n}$ & $\begin{array}{l}\text { Components of the linearized strain tensor in the basis } \\
\left\{\hat{\mathbf{e}}_{1}^{i}, \hat{\mathbf{e}}_{2}^{i}, \hat{\mathbf{e}}_{3}^{i}\right\}\end{array}$ \\
\hline$E_{i}$ & Young's modulus of the $i^{\text {th }}$ smooth fold region \\
\hline$\nu_{i}$ & Poisson's ratio of the $i^{\text {th }}$ smooth fold region \\
\hline$\Pi$ & Total potential energy \\
\hline$U$ & Elastic strain energy \\
\hline$W_{e}$ & $\begin{array}{l}\text { External work exerted by body forces and boundary trac- } \\
\text { tions }\end{array}$ \\
\hline$\Omega_{i}$ & Volume of the $i^{\text {th }}$ smooth fold region \\
\hline$z$ & Out-of-plane coordinate \\
\hline$h_{i}$ & Plate thickness of the $i^{\text {th }}$ smooth fold region \\
\hline$I_{i}$ & Area moment of inertia of the $i^{\text {th }}$ smooth fold region \\
\hline $\mathbf{f}^{i}$ & Force vector of the $i^{\text {th }}$ point load \\
\hline $\mathbf{u}^{i}$ & $\begin{array}{l}\text { Displacement vector of the point of application of the } i^{\text {th }} \\
\text { point load }\end{array}$ \\
\hline$N_{f}$ & Number of applied point loads \\
\hline $\mathcal{L}$ & Auxiliary function to the total potential energy \\
\hline $\mathfrak{R}$ & Residual vector \\
\hline g & Gravity vector \\
\hline$E_{A P}$ & Young's modulus of ABS plastic \\
\hline$E_{N F}$ & Young's modulus of NinjaFlex ${ }^{\circledR}$ \\
\hline$\nu_{A P}$ & Poisson's ratio of ABS plastic \\
\hline$\nu_{N F}$ & Poisson's ratio of NinjaFlex ${ }^{\circledR}$ \\
\hline$\rho_{A P}$ & Mass density of ABS plastic \\
\hline$\rho_{N F}$ & Mass density of NinjaFlex ${ }^{\circledR}$ \\
\hline
\end{tabular}

